Preprint: PRF/es2020apr22_717

DOI of published version: https://doi.org/10.1103/PhysRevFluids.5.083903

\title{
Wide domain simulations of flow over an unswept laminar wing
} section undergoing transonic buffet

\author{
Markus Zauner \\ Aerodynamics Aeroelasticity Acoustics Department, \\ ONERA - The French Aerospace Lab, Meudon 92190, FR \\ Neil D. Sandham \\ Aerodynamics and Flight mechanics Group, \\ University of Southampton, Southampton SO17 1BJ, UK
}

(Dated: August 2020) 


\begin{abstract}
Transonic buffet is an unsteady flow phenomenon that limits the safe flight envelope of modern aircraft. Scale-resolving simulations with span-periodic boundary conditions can provide detailed insight into the flow physics associated with buffet and can help to calibrate simplified models that are needed, for example, to develop more efficient wings based on laminar-flow supercritical sections. However, such simulations are often feasible only for severely restricted spanwise domains. In the current contribution, we analyse an unswept laminar-flow wing section (of Dassault Aviation's V2C profile) at a moderate Reynolds number of $R e=500,000$ and a Mach number of $M=$ 0.7 with spanwise domains equal to $5 \%$ and $100 \%$ of the airfoil chord. An implicit large-eddy simulation methodology, using a spectral error estimator to control the action of a high-order filter, is first validated against direct numerical simulations and then used for the domain width study. Quantitative differences, due to domain size, include an increase in amplitude and regularity of the buffet oscillations in the wider domain. Nevertheless, space-time analysis shows that key physical phenomena such as upstream-propagating shock waves are properly represented in the narrow domain and there is limited sensitivity to domain size of the aerodynamic coefficients. Even in the very wide domain, which is an order of magnitude wider than the largest turbulent structures measured at the trailing edge, certain features remain two-dimensional, including the shock and expansion waves that interact with the boundary layer upstream of transition. The transition mechanism is found to have subtle variations during a typical buffet cycle, with Kelvin-Helmholtz structures prominent during low-lift phases and oblique modes developing behind shock/boundarylayer interactions during high-lift phases. The availability of the wide-domain data is used for further study of the buffet mechanism, considering phase-averaged data and instantaneous flow fields to show the global structure of the buffet oscillation.
\end{abstract}

Keywords: CFD, transonic buffet, large eddy simulation, moderate Reynolds numbers

\footnotetext{
*mzauner@onera.fr Also at Aerodynamics and Flight mechanics Group, University of Southampton, Southampton SO17 1BJ, UK

m.zauner@soton.ac.uk

† n.sandham@soton.ac.uk
} 


\section{INTRODUCTION}

Transonic buffet is usually associated with large amplitude, autonomous shock oscillations, caused by the interactions between shock waves and separated shear layers, for which there is a substantial literature base [1 4]. Different types of shock motion (back and forth moving shocks or periodically upstream-propagating shock waves) have been studied by [5] and have also been reported for several rigid airfoils under buffet conditions [6 9]. The structural response of wings and control surfaces to these aerodynamic oscillations is commonly referred to as "buffeting", which limits the safe flight envelope of modern aircraft. Therefore, it is of great interest to be able to predict buffet onset as precisely as possible. While traditional explanations for transonic buffet (e.g. acoustic feedback and wave propagation models [8, 10 14]) have difficulties to directly couple the shock motion with the low-frequency fluctuations in the lift [15-17], more recent studies describe transonic buffet as a global instability [18 20].

Buffet has some different characteristics on swept versus unswept wings. While for unswept cases, transonic buffet is associated with a sharp spectral peak at Strouhal numbers (defined as $S t=f c / U_{\infty}$ ) in the range of $S t \approx 0.05-0.1$, a broadband frequency signature exists for swept-wing cases. Furthermore, chordwise shock oscillations are more confined in the swept cases with variations in the spanwise direction. Outboard-travelling shock waves can also be connected to so-called "buffet cells" [21 23]. Not everything is different, however, since recent studies for swept wings also exhibit a sharp spectral peak comparable to unswept cases [24], where the mode associated with the broadband swept-wing behaviour seems to be driven by flow-separation phenomena and converges towards a zero-frequency mode for decreasing sweep angles [25]. These results suggest that there is more to learn from unswept cases, as they not only capture phenomena that are still not fully understood, but are connected to more general swept-wing configurations.

So far, airfoils and wing sections with mainly turbulent boundary layers have dominated transonic buffet research. However, challenging emission goals have encouraged research into laminar-flow designs with potential fuel savings of the order of $10 \%$ for typical commercial aircraft. The key to this technology is to maintain laminar boundary layers over a large part of the airfoil in order to reduce skin-friction drag, even though laminar boundary layers can be more sensitive to disturbances and off-design conditions. To exploit its full potential it 
is important to understand and eventually to be able to control key flow phenomena such as laminar shock-wave/boundary-layer interaction and undesired instabilities like transonic buffet. Experimental studies have reported significant differences in the buffet characteristics under laminar flow conditions. For an RA16 airfoil with natural transition $(R e=3-6 \times$ $10^{6}$ ), [26] reported a strong increase of the pressure fluctuation level without any particular frequency, instead of the typical sharp spectral peak [27]. Also [28] compared laminar and turbulent buffet characteristics of an OAT15A airfoil and for the laminar case found a dominant peak at significantly higher frequencies $(S t \approx 0.9)$ compared to the turbulent case $(S t \approx 0.06)$. Numerical studies have recently been applied to laminar-flow airfoils. Analysing ONERA's OALT25 profile using large-eddy simulations, [29] reported a sharp spectral peak at significantly higher frequencies $(S t=1.2$ instead of $S t=0.06)$ and more confined shock motion compared to a case with tripped boundary layers. Experimental investigations on the same airfoil also reported this sharp peak at $S t=1.2$, but with a less dominant broadband phenomenon at $S t \approx 0.05$ [30]. Analyses of Dassault Aviation's laminar-flow V2C profile showed a quite different trend, as Strouhal numbers were reported in the range of $S t=0.12-0.16$ for laminar as well as for tripped turbulent cases, albeit different from typical Strouhal numbers mentioned above. This airfoil geometry was the subject of experimental [31, 32] as well as numerical studies [33 36] and is also considered for our investigations. It is likely that, over some parameter ranges and airfoil geometries, different types of buffet exist (or can even coexist) ([16, 30, 37]) and it is still unclear how they relate to each other and to turbulent cases [38].

Further progress to clarify the mechanisms can be made using scale-resolving simulations. However, due to the high resolution of direct numerical simulations (DNS) that is required even at moderate Reynolds numbers (for example grid spacings based on wall scaling of $\Delta x^{+}<5, \Delta y^{+}<1, \Delta z^{+}<5[37$, 39]), both the run time and domain width have been limited. For example, the grid required for a well-resolved DNS at moderate Reynolds numbers considering a narrow domain of $5 \%$ of the chord length contains approximately one billion grid points and required more than 130,000 core hours to compute one dimensionless time unit, which is defined as the ratio of chord length and freestream velocity $\left(c / U_{\infty}\right)$, making parametric studies unfeasible.

Large-eddy simulations (LES) have the potential to capture the main flow phenomena involved in buffet, saving several orders of magnitude in cost versus DNS. Previous 
scale-resolving simulations of airfoils at transonic buffet conditions have captured the lowfrequency unsteadiness [40] [41] [37], but have also recognised the sensitivity to the computational grid and sub-grid scale model [36, 40], which becomes even more of a challenge when details of the transition process must be captured [42]. The particular challenges include the need for stretched, curvilinear grids and the presence of significant portions of laminar boundary layer where any sub-grid model must turn off to avoid unphysical additional dissipation. In order to maintain the numerical stability of LES with sub-grid models, however, filtering is often required, and in an airfoil flow the amount of filtering necessary may be different within the boundary layer as opposed to the near acoustic field. Implicit LES can be attractive, since in this kind of flow one already has schemes designed for adding dissipation to capture shock waves without spurious oscillations [43]. However, without additional tuning, ILES can also be far too dissipative in key regions with transitional or turbulent flow features 44]. In this context, one aspect of the current work is to try to improve methods for airfoil flow and here we use a technique that is highly selective in where filtering is applied. In addition to shock capturing, which is carefully limited to the immediate vicinity of shock waves using a proven methodology, we also control filtering away from shocks, where mostly we are relying on central difference schemes that develop oscillations when the flow is under-resolved. The methodology adopted here is to detect these characteristic oscillations and apply local filtering, helping to minimise the amount of filtering. The method will be validated against DNS for a buffet test case. Eventually, the data of such benchmark cases can be used to improve turbulence and transition models in order to facilitate more efficient simulation methods (e.g. URANS) with increased complexity of the test case (e.g. half-wing configuration, fluid/structure interaction, etc.).

The main objective of the present contribution is to assess the influence of the spanwise domain size on key physical phenomena associated with buffet. If key phenomena are captured in the narrower domain, this justifies its use for further fundamental studies including instability analyses. If significant differences are found, then we know that we need to use the wider domains. To check the effects, we run two cases with a factor of 20 different spanwise domain size. In section [I] we describe the SE-ILES methodology, which is validated against DNS in section III, demonstrating excellent results with a factor of 16 overall saving in computer time relative to the DNS. In section [V] we make the central comparison of domain size using aerodynamic coefficients, $x / t$ diagrams and two-point correlations. In section $\mathrm{V}$ 
we exploit the data from the wide domain size to study the transition and low-frequency phenomena in more detail and make conclusions in section VI.

\section{METHODOLOGY}

All simulations reported here were carried out using the high-order parallel multi-block finite difference in-house code SBLI. Details of the solver and previous applications can be found in [45], 46], and [47]. The basic set-up outlined in this section is the same as the direct numerical simulations in [37]. The dimensionless time-dependent compressible Navier-Stokes equations in three spatial dimensions can be written as

$$
\begin{array}{r}
\frac{\partial \rho}{\partial t}+\frac{\partial \rho u_{i}}{\partial x_{i}}=0, \\
\frac{\partial \rho u_{i}}{\partial t}+\frac{\partial \rho u_{i} u_{j}}{\partial x_{j}}=-\frac{\partial p}{\partial x_{i}}+\frac{1}{R e} \frac{\partial \tau_{i j}}{\partial x_{j}}, \\
\frac{\partial E_{t}}{\partial t}+\frac{\partial E_{t} u_{j}}{\partial x_{j}}=-\frac{\partial p u_{j}}{\partial x_{j}}+\frac{\partial q_{j}}{\partial x_{j}}+\frac{1}{R e} \frac{\partial\left(u_{i} \tau_{i j}\right)}{\partial x_{j}} .
\end{array}
$$

The pressure and heat flux are denoted by $p$ and $Q$, respectively, while $\tau$ denotes the stress tensor. The non-dimensionalisation scheme used to formulate the above equations is based on the freestream density $\rho_{\infty}^{*}$, velocity $u_{\infty}^{*}$ and temperature $T_{\infty}^{*}$, where the asterisk denotes dimensional quantities. The pressure $p$ is normalised by $\rho_{\infty}^{*} u_{\infty}^{* 2}$. The characteristic length is set according to the airfoil chord length $c$. The five conservative variables are contained in a solution vector $q$, consisting of the density $\rho$, the specific momentum components $\rho u, \rho v$ and $\rho w$, and the volume specific total energy $E_{t}=\rho e_{i n t}+1 / 2 \times \rho(u u+v v+w w)$, where $e_{\text {int }}=T /\left(\gamma(\gamma-1) M^{2}\right)$ denotes the specific internal energy. The fluid is considered as a Newtonian fluid with the stress tensor

$$
\tau_{i j}=\mu\left(\frac{\partial u_{i}}{\partial x_{j}}+\frac{\partial u_{j}}{\partial x_{i}}-\frac{2}{3} \frac{\partial u_{k}}{\partial x_{k}} \delta_{i j}\right),
$$

where $\mu$ and $\delta_{i j}$ denote the kinematic viscosity and the Kronecker delta, respectively. The heat conduction is considered according to Fourier's law, written in dimensionless form as

$$
q_{j}=-\frac{\mu}{(\gamma-1) \operatorname{Re} \operatorname{Pr} M^{2}} \frac{\partial T}{\partial x_{j}}
$$

where specific heat ratio and Prandtl number are assumed to be constant with $\gamma=1.4$ and $\operatorname{Pr}=0.72$, respectively. 
(a)

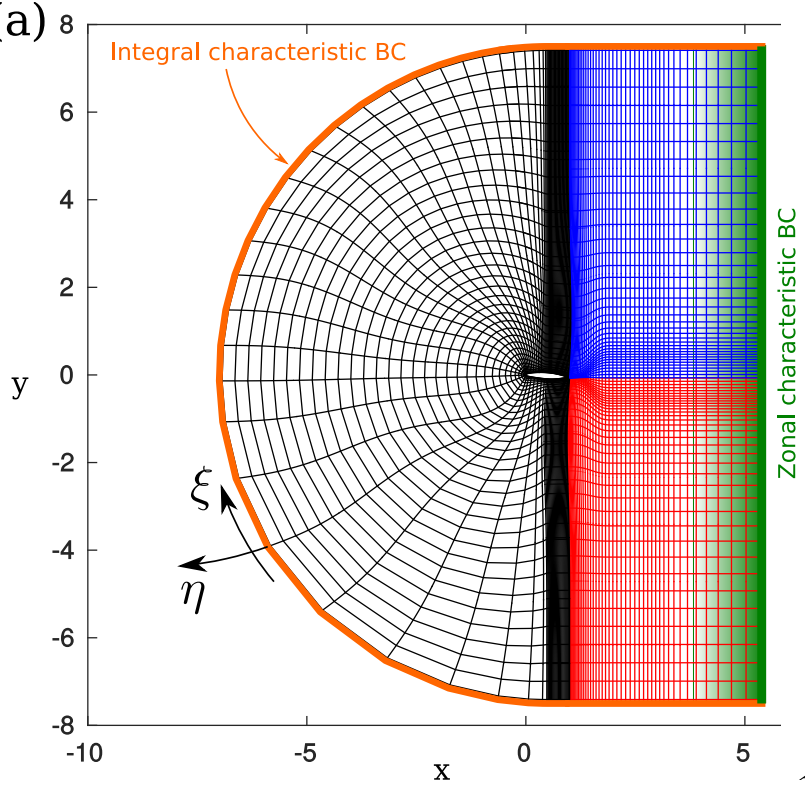

(b)

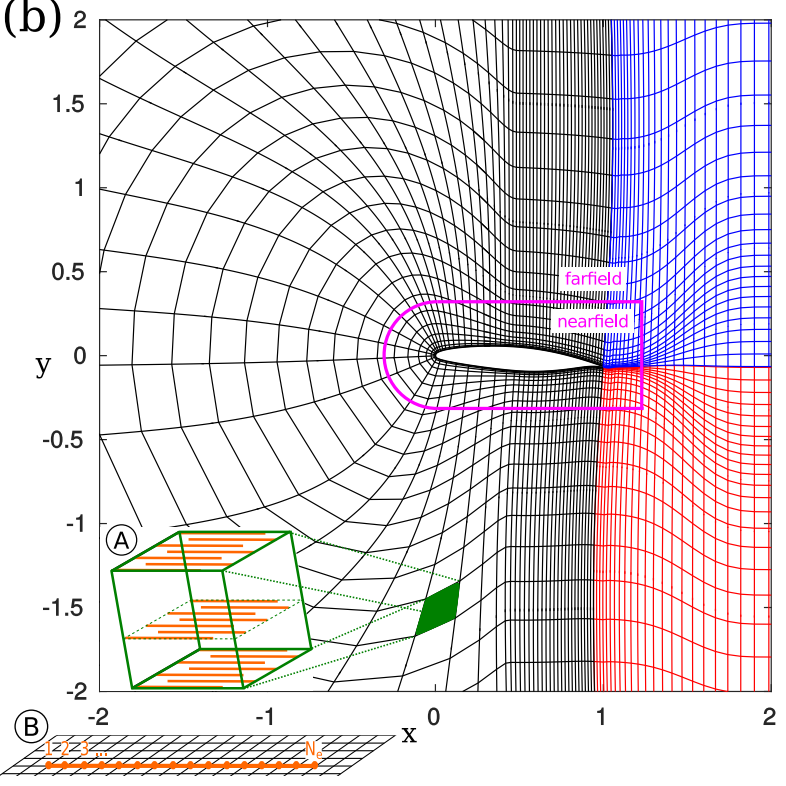

FIG. 1. (a) Multiblock LES grid plotting every $18^{\text {th }}$ and $14^{\text {th }}$ grid point in the $\xi$ - and $\eta$-direction, respectively. Integral characteristic and zonal characteristic boundaries are highlighted orange and green, respectively. (b) Close-up of the region around the airfoil, where the magenta lines sketch the boundary between near and far field. Details (A) and (B) indicate a processor block and a stencil for error indicator calculation, respectively.

The temperature dependency of the dynamic viscosity is modelled by Sutherland's law according to

$$
\mu=T^{\frac{3}{2}} \frac{1+C_{\text {Suth }}}{T+C_{\text {Suth }}},
$$

where the Sutherland constant is set to $C_{\text {Suth }}=110.4 / T_{\infty}^{*}=0.41$ with $T_{\infty}^{*}=268.67 \mathrm{~K}$. For statistical analysis, the Favre (density weighted) average of a variable $q$ is defined by

$$
\tilde{q}=\frac{\overline{\rho q}}{\bar{\rho}}
$$

where an overbar denotes the usual Reynolds average.

The equations are discretised in time using an explicit third-order low-storage RungeKutta scheme and in space by applying a fourth-order central finite difference scheme on a structured multi-block grid. Five-point stencils are used to calculate the interior of the flow field, while the boundaries are treated by a fourth-order accurate scheme according to [48]. The same discretisation schemes were used for DNS of airfoil flows by [47]. Figure 1 shows 
(a) an LES grid (every $18^{\text {th }}$ and $14^{\text {th }}$ grid point in the $\xi$ - and $\eta$-direction, respectively) and (b) a close-up of the airfoil (details (A) and (B) will be explained later). Zonal characteristic boundary conditions [49] are applied at the outlet (highlighted with green in figure 1(a)), whereas integral characteristic boundary conditions [50] are applied at the remaining outer boundaries (highlighted in orange). Due to the appearance of shock waves, a total variation diminishing (TVD) scheme is used, but limited to the immediate vicinity of shock waves and turned off completely within a small zone near the leading edge, as described in 37. To avoid the formation of spurious structures in the freestream, a sixth-order filter, similar to the one used by [51] is incorporated in the farfield, but disabled within a distance of $\Delta n \approx 0.2$ from the airfoil surface (the boundary is sketched by the magenta lines), so that the boundary layer and near-wake flow features are not affected. For the filtering, we solve the tridiagonal system

$$
\alpha_{f} \breve{q}_{(i-1)}+\breve{q}_{i}+\alpha_{f} \breve{q}_{(i+1)}=\sum_{n=0}^{N} \frac{a_{n}}{2}\left(q_{(i+n)}+q_{(i-n)}\right) \text { with } N=3,
$$

where $q$ and $\breve{q}$ denote unfiltered and filtered components of the solution matrix, respectively. The adjustable parameter is set to $\alpha_{f}=0.45$, while the coefficients are derived in terms of $\alpha_{f}$ using Taylor- and Fourier-series analyses [51] as

$$
a_{0}=\frac{11}{16}+\frac{5 \alpha_{f}}{8}, a_{1}=\frac{15}{32}+\frac{17 \alpha_{f}}{16}, a_{2}=\frac{-3}{16}+\frac{3 \alpha_{f}}{8}, a_{3}=\frac{1}{32}+\frac{1 \alpha_{f}}{16} .
$$

[52] and [53] provide more detailed information on the filtering technique. The filter is applied to the conserved variables in each spatial direction in a sequential way. In the current implementation, an additional coefficient $a_{l i m}$ is specified according to the update algorithm

$$
q_{\text {new }}=q_{\text {orig }}-a_{\text {lim }} \cdot\left(q_{\text {orig }}-\breve{q}\right),
$$

where $q_{\text {orig }}$ is the solution before applying the filter and $q_{\text {new }}$ contains the updated variable. For direct numerical simulations, $a_{l i m}$ is set to $a_{l i m}=0.1$ in the freestream region, where the filter is applied. This approach has also been used to improve the numerical stability of simulations with decreased resolutions, for example during initial grid studies. More details on the numerical implementation are provided in [37].

The present contribution utilises a newly-developed spectral-error based implicit largeeddy simulation (denoted SE-ILES) approach, which uses the same $6^{\text {th }}$-order filter as used 
in the freestream, but with the filter coefficient set to $a_{l i m}=0.4$. In the SE-ILES approach, this filter is only applied in regions that are developing grid-to-grid point oscillations, and hence does not affect structures in well-resolved regions. Corresponding to the number of available computational cores, the domain is split into subdomains, here denoted as processor blocks $(\mathrm{PBs})$, so that each core solves the flow for approximately the same number of grid points. In the present case, each three-dimensional PB contains approximately $\sim 25,000$ grid points ( $\sim 2500$ grid points in the $2 \mathrm{D}$ plane) and sketched by the green box (detail (A)) in figure 1(b). To identify insufficiently resolved regions of the flow we adopt a spectral error estimator previously used to identify regions of poor resolution and then for iterative grid design [54, 55]. For fully-developed turbulence we expect the energy in small-scale structures to reduce with increasing wavenumber according to Kolmogorov's $-5 / 3$ law in the inertial sublayer, or at an even higher rate in the dissipation range. However, for high-order finite difference schemes, we often observe significantly weaker decay rates, or even spectra that increase with increasing wavenumber, in regions of reduced grid resolution. Spectral error indicators are used to efficiently estimate the actual spectral decay rate against a specified minimum acceptable decay rate. Explicit filtering is then applied only in regions with high error severity. Spectral error indicators are also applied in the context of spectral element simulations of wing flows to control grid adaptation [56].

In the present approach, spectral error indicators are calculated every $N_{E}=10$ time steps for each processor block according to [54] using the vorticity magnitude $|\omega|$. Instead of computing the full spectra for each $3 \mathrm{D} \mathrm{PB}$, the Fourier amplitudes $A_{2}, A_{4}$, and $A_{8}$ (proportional to the square root of the spectral energy) of selected modes $\left(N_{e} / 2, N_{e} / 4\right.$, $N_{e} / 8$ ) for stencils of $N_{e}=16$ grid points (detail (B) in figure 1(b)) are defined by

$$
\begin{array}{r}
A_{2}=\frac{2^{-2 r}}{N_{e}} \sum_{j=0}^{N_{e}-1}(-1)^{j}\left|\omega_{j}\right|, \\
A_{4}=\frac{2^{1-r}}{N_{e}} \sum_{j=0}^{N_{e}-1}(-i)^{j}\left|\omega_{j}\right|, \\
A_{8}=\frac{2}{N_{e}} \sum_{j=0}^{N_{e}-1} \exp \left(-\frac{\pi}{4} i\right)^{j}\left|\omega_{j}\right| .
\end{array}
$$

The parameter $r$ denotes the minimum acceptable decay rate of amplitudes in the shortwavelength part of the spectrum, here set to $r=-1$. The average of Fourier amplitudes $(\bar{A})$ of a set of similarly-oriented stencils, which are homogeneously distributed within the 
PB (indicated by the staggered orange lines for representative planes in detail (A) of figure $1(b))$, is calculated and the mean error indicator $\overline{I_{f}}$ is obtained by

$$
\begin{array}{r}
\overline{I_{f}}=\ln \left(1+\left\lfloor\frac{\overline{A_{2}}}{\overline{A_{4}}+\epsilon}\right\rfloor+\left\lfloor\overline{\overline{A_{4}}}\right\rfloor+\left\lfloor\frac{\overline{A_{2}}}{\overline{A_{8}}+\epsilon}\right\rfloor\right), \\
\text { with } \overline{\mathrm{A}_{2}}=\sum_{\mathrm{n}=1}^{\mathrm{N}_{\text {stencil }}} \frac{\mathrm{A}_{2, \mathrm{n}}}{\mathrm{N}_{\text {stencil }}}, \overline{\mathrm{A}_{4}}=\sum_{\mathrm{n}=1}^{\mathrm{N}_{\text {stencil }}} \frac{\mathrm{A}_{4, \mathrm{n}}}{\mathrm{N}_{\text {stencil }}} \text {, and } \overline{\mathrm{A}_{8}}=\sum_{\mathrm{n}=1}^{\mathrm{N}_{\text {stencil }}} \frac{\mathrm{A}_{8, \mathrm{n}}}{\mathrm{N}_{\text {stencil }}},
\end{array}
$$

where $\lfloor\ldots\rfloor$ is a 'floor' operation and $\epsilon=0.03$ used to avoid division by zero and tune the error-indicator sensitivity [54]. In cases where $\overline{I_{f}}$ exceeds a value of $\varepsilon_{1}=0.6$, the error in this PB is considered as high. The threshold is based on a grid study of the Taylor-Green vortex test case [54]. In addition to the instantaneous error, an average error is computed according to

$$
\begin{array}{ll}
\varepsilon_{a v}=\left(1-C_{a v}\right) \varepsilon_{a v}+C_{a v} 1 & \text { if } \overline{\mathrm{I}_{\mathrm{f}}}>\varepsilon_{1}, \\
\varepsilon_{a v}=\left(1-C_{a v}\right) \varepsilon_{a v}+C_{a v} \frac{N_{\text {high }}}{N_{\text {check }}} & \text { else, }
\end{array}
$$

which indicates the probability of $\overline{I_{f}}$ exceeding the threshold $\varepsilon_{1}$ in this PB using $C_{a v}=0.1$. The number of cases in which spectral errors were calculated and exceeded the threshold $\varepsilon_{1}$ is denoted by $N_{\text {high }}$, while $N_{\text {check }}$ denotes the total number of checks.

Every $N_{F}=30$ time steps, the filter is applied in a $\mathrm{PB}$, if either $\overline{I_{f}}>\varepsilon_{1}$ or $\varepsilon_{a v}>\varepsilon_{2}$ (for the present study $\varepsilon_{2}=0.2$ ). Blending is used to transition from $a_{l i m}=0.4$ (in PBs with an high spectral error) to $a_{\text {lim }}=0.0$ in adjacent PBs with low error estimates. This is repeated for three stencil sets within each PB to calculate error indicators corresponding to the curvilinear coordinates and to apply the filter in each direction independently.

Table \summarises relevant parameters for the spectral-error based ILES approach. Parameters $r, N_{e}, \epsilon, \epsilon_{1}$ are based on [54], where these settings were shown to do a good job of capturing grid-to-grid point oscillations. The remaining parameters were tuned in the current work and the methodology is applied throughout the simulations. Calculating spectral errors every $N_{E}=10$ time steps corresponding to $3.2 \times 10^{-4}$ time units was found to be sufficient to gain a good representation of the average error $\epsilon_{a v}$, when applying filtering (if required) every $N_{F}=30$ time steps. In general, it is recommended to keep $N_{E}<N_{F}$ to avoid an underestimation of the errors. The factor $N_{F}$ was adopted from previous uses of filtering to enhance numerical stability. Factor $\epsilon_{2}$ depends on $N_{E}$, as it accounts for fluctuating error-indicator values within the filter intervals and can be used to increase the 
TABLE I. Overview of spectral-error based filter parameters.

\begin{tabular}{|c|c|c|c|c|c|}
\hline \multicolumn{2}{|c|}{ Computation of SE indicators } & \multicolumn{4}{|c|}{ Control of filter activity } \\
\hline$r \quad N_{e} \epsilon$ & $N_{E}$ & $N_{H}$ & $a_{\text {lim }}$ & $\epsilon_{1}$ & $\epsilon_{2}$ \\
\hline $\begin{array}{lll}-1 & 16 & 0.03\end{array}$ & 10 & 30 & 0.4 & 0.6 & 0.2 \\
\hline
\end{tabular}

sensitivity. The parameter $a_{l i m}$ can be used used to control numerical stability. However, if this parameter becomes too high it is better to adapt the grid resolution instead. More details on spectral error-indicators and the chosen settings can be found in [54] and [55], respectively.

\section{COMPARISON OF NARROW-DOMAIN DNS AND LES}

To validate the SE-ILES approach, comparisons are made with DNS. The objective is to check that various phenomena associated with buffet can be reproduced using the cheaper approach. This then enables longer run times and simulations with wider domains.

Previous DNS were reported in [37]. These were run at a moderate Reynolds number of $R e=500,000$, a Mach number of $M=0.7$, and an angle of attack of $\alpha=4^{\circ}$. An extensive grid study resulted in a choice of a computational grid for this flow configuration with around one billion grid points, based on a spanwise domain width equal to $5 \%$ of the airfoil chord. Due to the computational cost, only a short run time of four buffet cycles was possible, but this is sufficient to assess the capability of SE-ILES to capture the main phenomena.

A summary of the simulations discussed in this section is given in table II] listing the angle of attack $\alpha$, Reynolds number, grid size, spanwise domain extent $L_{z}$, number of grid points in the spanwise direction $N_{z}$, grid spacing of the smallest cells $\left(\Delta x_{\min }\right.$ and $\left.\Delta y_{\min }\right)$, and $6^{\text {th }}$-order filter activity. In the DNS the filtering is only applied in the farfield, where the grid stretching results in under-resolved acoustic waves, whereas in the SE-ILES filtering is applied in those blocks where under-resolution is detected, as described in the previous section. The DNS was started from a 2D solution at a lower Reynolds number, which was extruded in the spanwise direction. Initial volume forcing similar to [47] was added to the right-hand side of the momentum equations to introduce a random three-dimensional 
TABLE II. Overview of simulations discussed in section III.

\begin{tabular}{llcccccccc}
\hline Case & Name & $\alpha$ & Re & Grid points & $L_{z}$ & $N_{z}$ & $\Delta x_{\text {min }}$ & $\Delta y_{\text {min }}$ & Filter \\
\hline$A 0$ & DNS & $4^{\circ}$ & $500 \times 10^{3}$ & $1.07 \times 10^{9}$ & $0.05 c$ & 150 & $4 \times 10^{-4}$ & $6.8 \times 10^{-5}$ & farfield \\
$B 0$ & SE-ILES & $4^{\circ}$ & $500 \times 10^{3}$ & $75.3 \times 10^{6}$ & $0.05 c$ & 50 & $1 \times 10^{-3}$ & $1.7 \times 10^{-4}$ & targeted \\
\hline
\end{tabular}

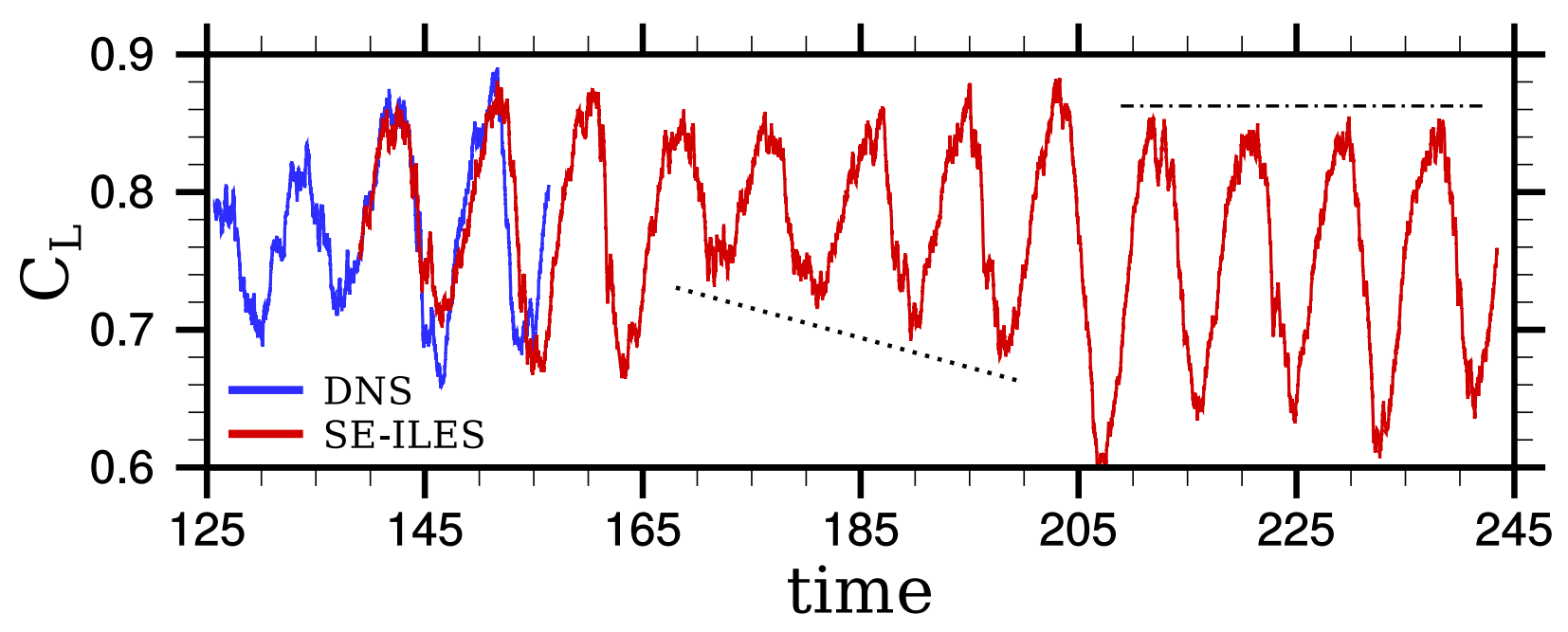

FIG. 2. Instantaneous lift coefficient (of a 2D-slice at $z=0$ ) of a DNS (blue curve) and SE-ILES (red curve) as a function of time.

disturbance at the leading edge for a short time period only, which accelerated the formation of turbulent structures. After self-sustained turbulence was fully developed on both sides of the airfoil, the Reynolds number was increased in a stepwise manner, until the target $R e=500,000$ was reached. To initialise the SE-ILES, a DNS solution taken after a few buffet cycles was interpolated onto the coarser SE-ILES grid. It should be noted that starting a SE-ILES with the same set-up from a uniform flowfield as an initial condition instead, would still develop the same low-frequency unsteadiness, but leads to a longer transient of at least two low-frequency cycles.

Figure 2 shows the lift coefficient as a function of time for the DNS (blue curve) and SE-ILES (red curve), both with a spanwise domain extent of $5 \%$ of the chord length. Looking at the DNS results, low-frequency fluctuations are observed at a Strouhal number of $S t \approx 0.12$. The amplitude of the $C_{L^{-}}$oscillation shows some variation, with the last two cycles having larger amplitudes than the first two. Similar variability can be seen in the 
SE-ILES results. Shortly after restarting from the DNS solution, the SE-ILES develops a low-frequency phenomenon with similar amplitudes to the DNS. The oscillation is irregular: after approximately 32 time units the minimum lift increases and then steadily decreases again (indicated by the black dotted line). After another four low-frequency cycles, there is

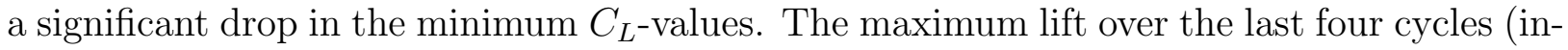
dicated by the black dash-dotted line in figure 2 ) is reasonably constant, while the minimum values still show significant variations. Over the full SE-ILES run-time the variation of the maxima is only $\Delta C_{L} \approx 0.03$, while for the minima it is $\Delta C_{L} \approx 0.14$. The effect of spanwise domain size on this behaviour will be shown in the next section. Since a turbulent flow is present, it is expected that the SE-ILES and DNS solutions will eventually diverge from one another. In the context of this paper, the initial agreement between DNS and SE-ILES, with the SE-ILES closely matching the DNS over the first two cycles suggests that the key physical mechanisms resulting buffet are captured in the SE-ILES.

A more detailed comparison of DNS with SE-ILES can be made in the context of $x / t$ diagrams that have been used previously [37] to show the shock and boundary-layer phenomena associated with key frequencies visible in energy spectra. For orientation, figure 3(a) shows an instantaneous 2D slice at $z=0$ of the streamwise pressure gradient of the DNS at $t=156.4$. Red contours correspond to compressions, including shock waves, while blue lines indicate expansion regions. It can be seen that transition to turbulence occurs towards the rear of the airfoil, on both the suction and pressure surfaces, while shock and expansion waves are present upstream, above laminar boundary layers. The white dashed curve, located at a fixed distance $(\Delta n=0.05 c)$ from the airfoil surface is used to monitor and store time-resolved flowfield data, whereas the green lines denote sonic lines $(M=1)$.

Figures $3(\mathrm{~b}-\mathrm{c}$ ) show contours of streamwise pressure gradient $\partial p / \partial x$ as a function of space $(x)$ and time $(t)$ for the narrow domain (b) DNS and (c) SE-ILES along the monitor curve defined in part (a) of the figure. The green lines denote sonic lines and indicate the path of shock waves intersecting the monitor plane. It can be seen that these waves generally move upstream up to about the $30 \%$ chord location. The blank region at $t \approx 163$ in figure 3 (c) is omitted due to corrupted data, but the trends are clear in this region. In addition, the vertical white lines visible near the leading edge are an artifact of the post-processing which took the closest point to $\Delta n=0.05$. These lines are less pronounced in figure 3(a) due to the increased resolution of the DNS. On the right hand side of the contour plots, the 


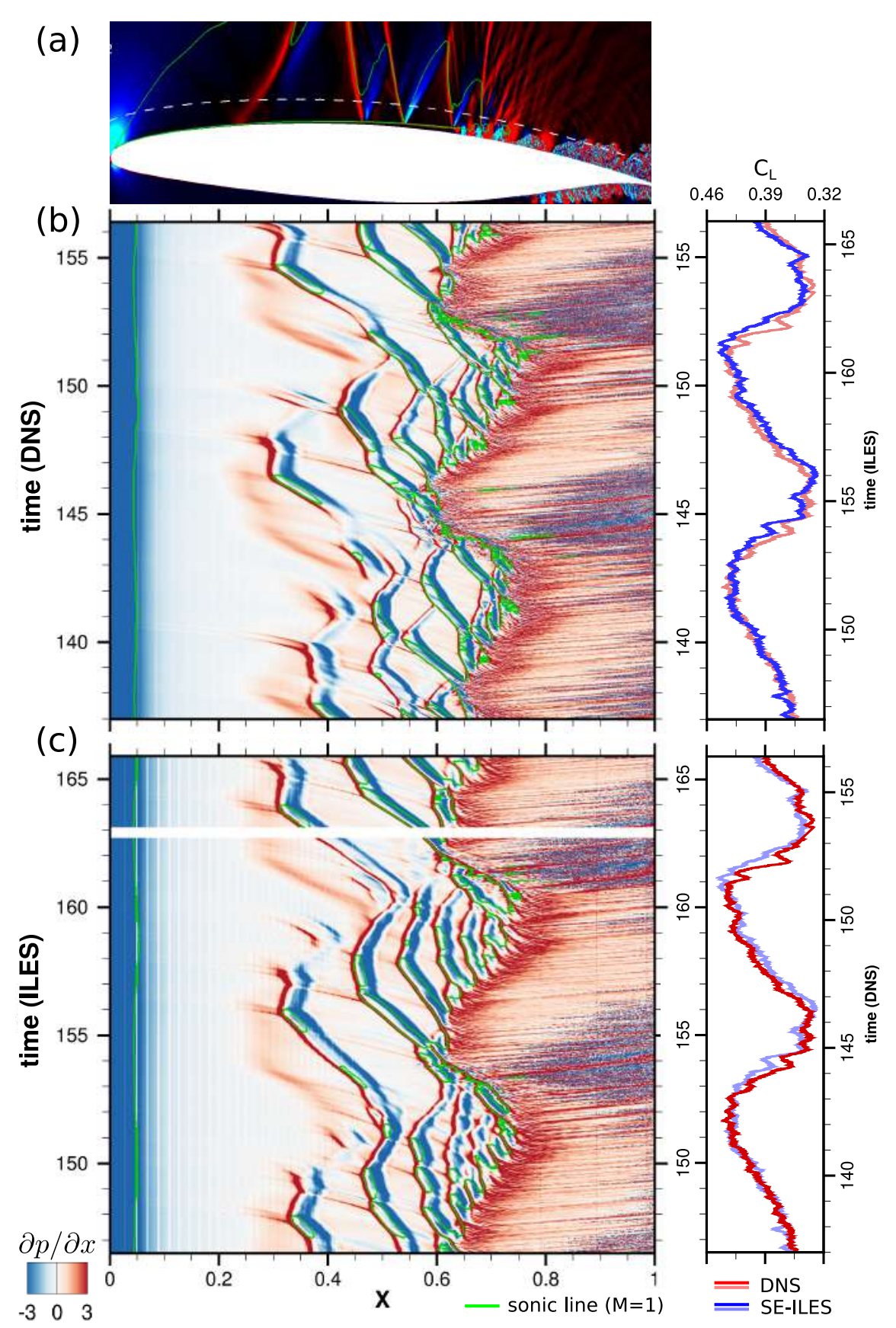

FIG. 3. (a) Snapshot showing contours of the streamwise pressure gradient of the DNS at $t=156.4$. Contours of the streamwise pressure gradient of the narrow-domain (b) DNS and (c) SE-ILES as a function of $x$ and time along the white dashed curve in (a). Green curves denote the sonic lines and the lift coefficient is shown as a function of time for DNS (blue) and SE-ILES (red) on the right-hand side of the $x / t$ diagrams. Please note that the time intervals are chosen to synchronise low-lift and high-lift phases of both simulations. 
lift coefficient is shown as a function of time. Representative time segments of SE-ILES and DNS were chosen and synchronised according to their low-frequency cycles. It can be seen that the lift oscillation corresponds to large scale forward and backward motion of the line of intermittent separation of the boundary layer, which moves forward to $60 \%$ chord during low-lift parts of the cycle and backwards to $70 \%$ chord during high lift parts of the cycle. The separation is followed by fine lines with positive slope. These lines initially correspond to the convection of Kelvin-Helmholtz roll-ups of the separated shear layer. Further downstream the lines are further apart and correspond to large-scale turbulent flow features, convecting over the airfoil surface. During low-lift phases, the large vortices appear as noisy patches near the trailing edge, showing up here because their influence reaches up to the white-dashed monitor line in figure $3(\mathrm{a})$.

Further interesting details of the flow can be observed in figures $3(\mathrm{~b})$. At $x \approx 0.05$, the green line indicates the beginning of the supersonic region. In the present case, shock trains are found in the region from $x=0.3$ to $x=0.6$ and usually consist of repeating patterns of a compression wave (red contours in figures $3(\mathrm{~b})$ ), followed by a subsonic region and an expansion fan (blue contours in figure 3(b)) turning the flow and returning it to a supersonic condition. The shock and expansion waves form a V-shaped structure in the $x y$-plane, which is well pronounced around the half-chord location in figure 3(a). Where the compression waves meet the surface there is a thickening of the boundary layer due to shock-wave/boundary-layer interaction, which can be observed in figure 3 (a) at $x \approx 0.3$. On the other hand, in the same figure, acoustic waves generated downstream of the supersonic region propagate upstream, slow down when approaching the supersonic region, where they strengthen and merge to form new shock waves.

All these flow features are remarkably consistent between the DNS version in figure $3(\mathrm{~b})$ and the SE-ILES version in figure 3(c). None of the described phenomena are significantly different in the two figures, allowing us to have confidence that the SE-ILES are properly capturing the flow physics and can be used for further parametric studies. After having validated the SE-ILES method against a DNS, we will compare next the SE-ILES of this section with simulation considering a much wider computational domain. 
TABLE III. Overview of simulations discussed in section IV.

\begin{tabular}{llcccccc}
\hline Case & Name & $\alpha$ & $R e$ & Grid points & $L_{z}$ & $N_{z}$ & Filter \\
\hline$B 0$ & SE-ILES narrow & $4^{\circ}$ & $500 \times 10^{3}$ & $75.3 \times 10^{6}$ & $0.05 c$ & 50 & targeted \\
$C 0$ & SE-ILES wide & $4^{\circ}$ & $500 \times 10^{3}$ & $1.5 \times 10^{9}$ & $1 c$ & 1000 & targeted \\
\hline
\end{tabular}

\section{EFFECT OF SPANWISE DOMAIN SIZE}

In this section, we consider the impact of the spanwise domain extent on the buffet and related phenomena. It is of particular interest to confirm whether $2 \mathrm{D}$ phenomena, such as boundary-layer instabilities or shock waves reported by [37] (where studies were limited to $\left.L_{z} \leq 0.25\right)$ are preserved in much wider domains. We would like to be able to establish guidance for future DNS studies, by determining which phenomena can be safely studied in relatively narrow domains and which other phenomena, such as potentially highly-threedimensional separations, require much wider computational domains.

In this study, we use the same spectral-error based large-eddy simulation (SE-ILES), which was validated in the previous section and compare it to a corresponding simulation considering a 20 times wider domain $\left(L_{z}=1 c\right)$. The details of the simulations are summarised in table III as case $C 0$.

An initial comparison of the effect of the massively increased computational domain is shown by the lift coefficient on figure 4. The solid black line shows the wide-domain SE-ILES (case $C 0$ in table III) as a function of time, while the red line shows the narrow domain case ( $B 0$ from the previous section). To initialise the wide-domain SE-ILES, a narrow-domain SE-ILES solution was periodically replicated in the spanwise direction and left to evolve lower spanwise wavenumbers. This transient phase of the wide-domain SE-ILES is shown by the dashed grey line in figure 4. For case $C 0$, representative time-instances at high-lift and low-lift phases are labelled WH and WL, respectively, and will be discussed later with respect to figure 11 .

Two observations can be made immediately from figure 4. Firstly, the amplitude of lift oscillations increases significantly in the wider domain and secondly, the oscillations become significantly more regular. As shown later in $3 \mathrm{D}$ visualisations of section $\mathrm{VB}$, the wide-domain case exhibits larger vortical structures. The increased energy content captured 


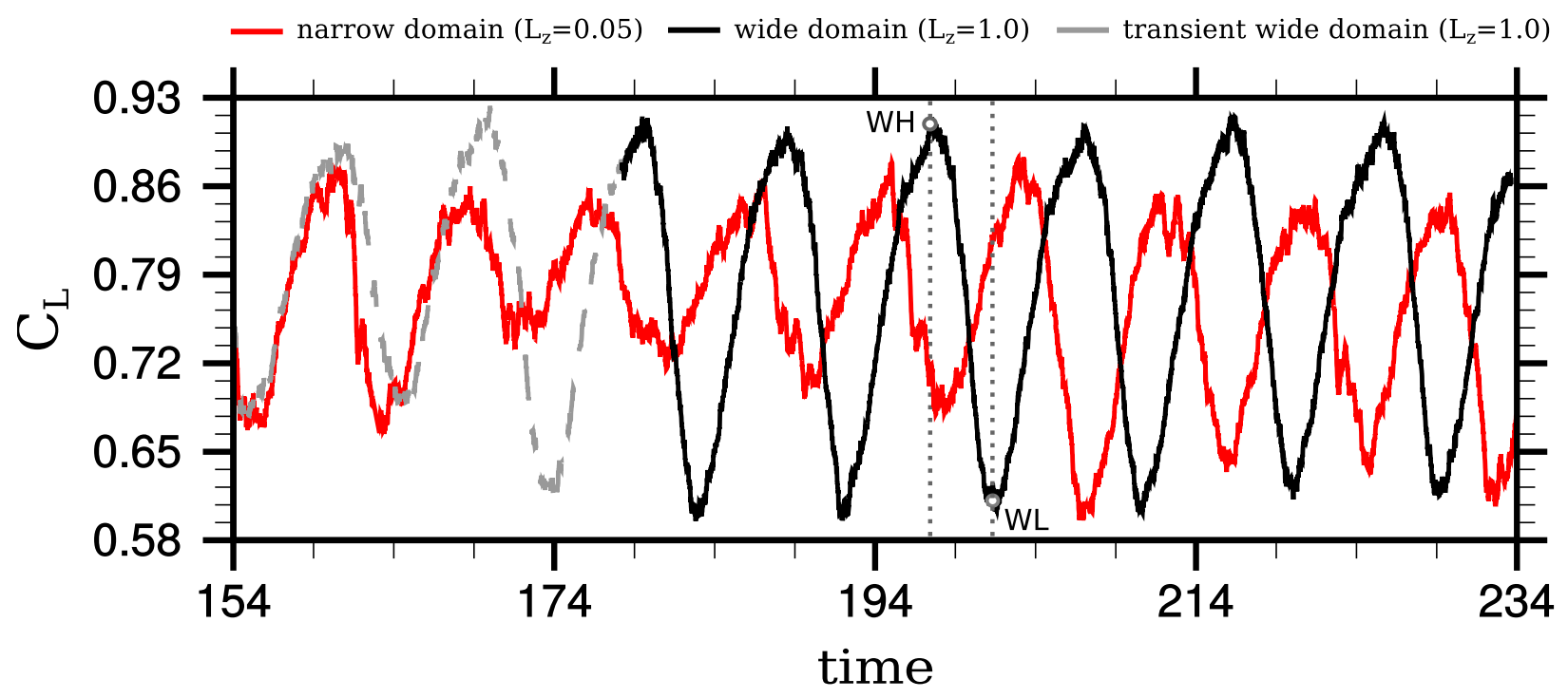

FIG. 4. Lift coefficient as a function of time for SE-ILES considering wide (black solid line) and narrow (red solid line) domains. The dashed-grey line denotes the transient phase of the wide SE-ILES. Labels $W h$ and $W L$ correspond to snapshots in figure 11 .

TABLE IV. Aerodynamic coefficients. Values in bracket denote the percental deviation from the wide-domain case.

\begin{tabular}{lcccccc}
\hline Name & $C_{L} / C_{D}$ & $C_{L}$ & $C_{D}$ & $C_{D, f}$ & $C_{D, p}$ & $\left.C_{D, p}\right|_{T E}$ \\
\hline SE-ILES wide & $20.28( \pm 0 \%)$ & $0.77( \pm 0 \%)$ & $0.038( \pm 0 \%)$ & 0.0038 & 0.034 & -0.014 \\
SE-ILES narrow & $20.97(+3 \%)$ & $0.76(-1 \%)$ & $0.036(-5 \%)$ & 0.0037 & 0.032 & -0.014 \\
\hline
\end{tabular}

by the wider domain seems to be the reason for increased amplitudes of lift oscillations. Despite the differences in unsteady lift coefficients, mean values of aerodynamic coefficients summarised in table IV agree well $( \pm 5 \%)$ for the wide- and narrow-domain cases. For example the lift coefficient $C_{L}$ only changes by $1 \%$ and the lift-to-drag ratio only changes by $3 \%$. The drag coefficient $C_{D}$ in table IV is decomposed into skin-friction and pressure drag components according to $C_{D, f}$ and $C_{D, p}$, respectively. The contribution of the base pressure at the blunt trailing edge is denoted by $\left.C_{D, p}\right|_{T E}$ in table IV. The mean trailing-edge pressure, which is often assumed to play an important role for buffet-related phenomena, does not seem to be affected by the domain size. 


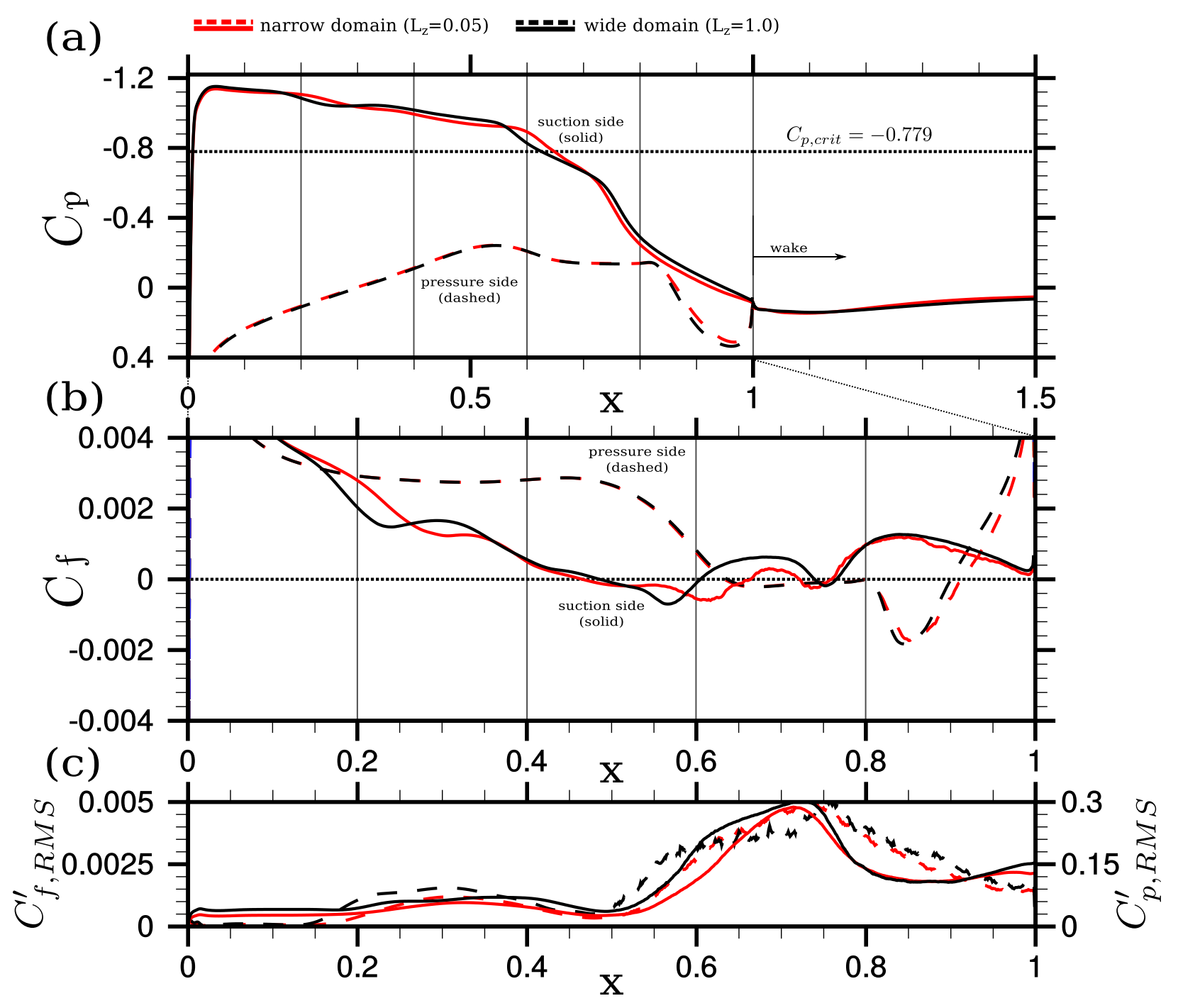

FIG. 5. Time- and span-averaged (a) pressure, (b) skin-friction coefficient, and (c) root-meansquare of corresponding fluctuations on the suction side as a function of chord position $x$ for narrow (red) and wide domain (black) cases. Critical pressure coefficient $C_{p, c r i t}=-0.779$ is indicated by the horizontal dotted line. In (a) and (b), solid and dashed lines correspond to suction and pressure sides, respectively, while solid and dashed lines in (c) denote $C_{p, R M S}^{\prime}$ (right-hand-side scale) and $C_{f, R M S}^{\prime}$ (left-hand-side scale), respectively.

To analyse the variations observed in integral quantities in more detail, figure 5 shows time-averages of (a) wall-pressure coefficient $C_{p}$ and (b) skin-friction coefficient $C_{f}$ as a function of chord position $x$. The solid and dashed lines correspond to suction side (SS) and pressure side (PS), respectively. The red line corresponds to the narrow domain, while 
the black line shows the wide domain. The dotted horizontal line in figure 5(a) indicates the critical pressure coefficient $C_{p, c r i t}$ (i.e. the pressure coefficient, where an isentropic flow would reach sonic conditions) defined as

$$
C_{p, \text { crit }}=\frac{2}{\gamma M^{2}}\left[\left(\frac{2+(\gamma-1) M^{2}}{\gamma+1}\right)^{\frac{\gamma}{\gamma-1}}-1\right],
$$

corresponding under the present flow conditions to $C_{p, c r i t}=-0.779$. The solid and dashed lines in figure 5(c) show the root-mean-square of the $C_{p}$ and $C_{f}$ fluctuations on the suction side, respectively. A general observation again is that the mean flow is not very sensitive to the spanwise domain size. The pressure distribution is almost completely unchanged, while the skin friction shows a slightly (5\%) earlier separation for the wide domain and the upper-side $C_{f}$ and $C_{f, R M S}^{\prime}$, appear earlier and stronger in the wide-domain case, consistent with the larger $C_{L}$ variations.

In order to get a better impression of the unsteady flow features for both cases, we consider $x / t$ diagrams for the narrow- and wide-domain cases, which are compared in figure 6. While the corresponding earlier figure 3(b) showed only a short time interval consisting of two low-frequency cycles, figure 6(a) shows contours of the streamwise pressure gradient for six buffet cycles. A corresponding $x / t$ diagram is shown in figure 6(b) for the wide-domain case. The general structure of the $x / t$ diagrams has been already discussed in the previous section in connection with figure 3. Comparing the wide- and narrow-domain cases we see that the patterns are more regular in the wide domain case. In addition we see a wider range of movement of the separation line in the wide domain case. Both observations are consistent with the changes seen in the lift time histories.

Given the greater regularity and repeatability of the wide domain simulation, it is worth exploring some of the features of the $x / t$ diagrams in more detail. On the left hand side of figure $6(\mathrm{~b})$ at $x \approx 0.05$, the green line indicates the front end of the supersonic region, oscillating at a frequency similar to the lift coefficient, but slightly phase shifted. This oscillation is caused by varying slope of the sonic line in the $x y$-plane near the front of the airfoil and is not affected by the domain width. Above the laminar region in the range $0.2<x<0.5$, the path of shock waves is well approximated by the black dotted lines in figure 6(b) corresponding to a constant speed of $u_{s}=-0.09$. Considering the speed of sound in the freestream $(a=1 / 0.7 \approx 1.43)$, the Mach number of the upstream-propagating shock waves $M_{s}=-u_{s} / a \approx 0.06$ is significantly lower compared to acoustic waves (sketched as a 

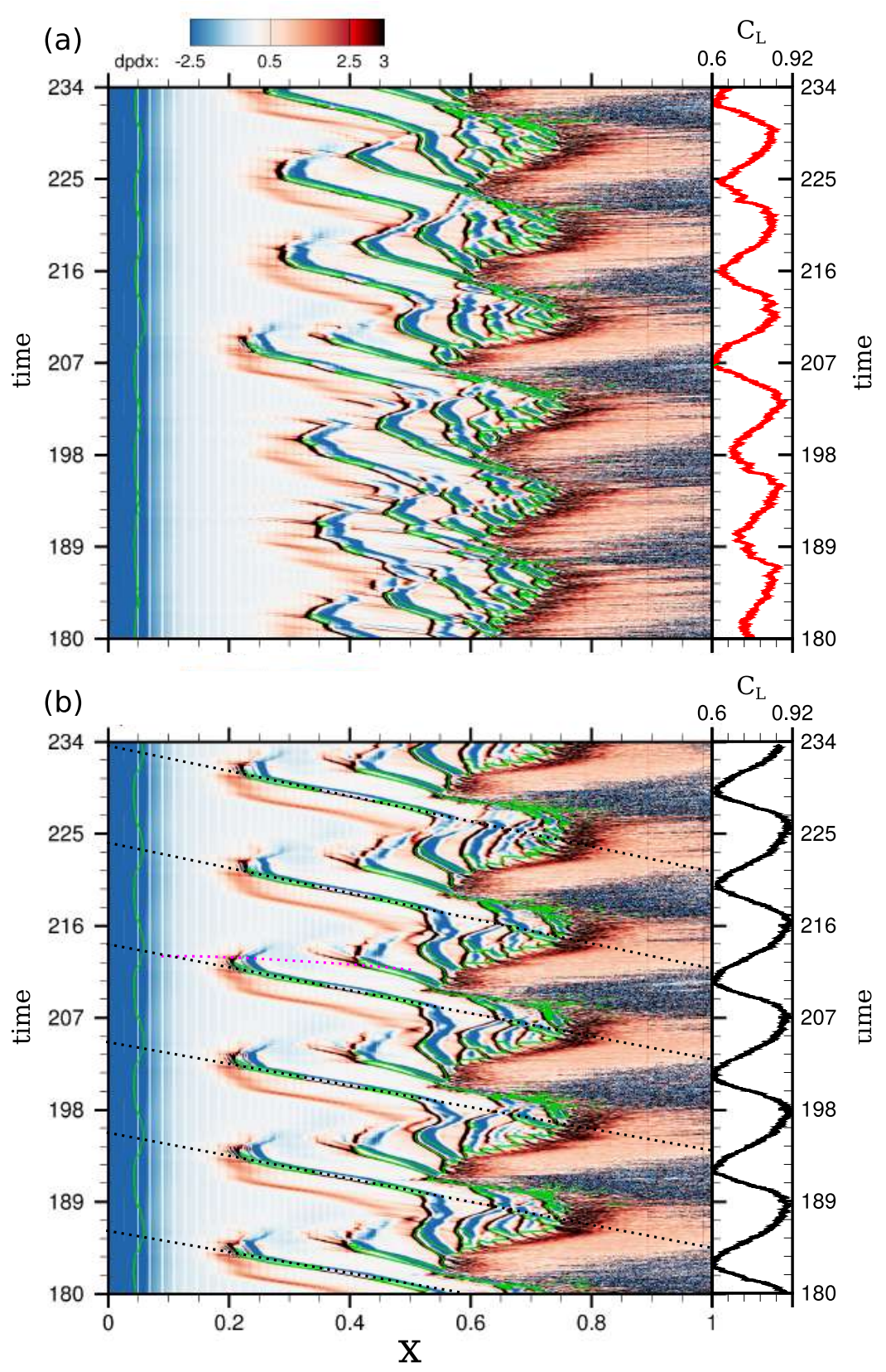

FIG. 6. Contour of the streamwise pressure gradient of the (a) narrow-domain and (b) widedomain SE-ILES as a function of $x$ and time at a distance of $\Delta y=0.05 c$ from the upper airfoil surface. Green curves denote the sonic lines and the lift coefficient is shown as a function of time for narrow- (red) and wide-domain SE-ILES (black) on the right-hand side of the $x / t$ diagrams. Traces of shock waves and acoustic waves are indicated by black-dotted and magenta-dash-dotted lines in (b), respectively. The lift coefficient is shown at the right hand side of the contour plots as a function of time. 


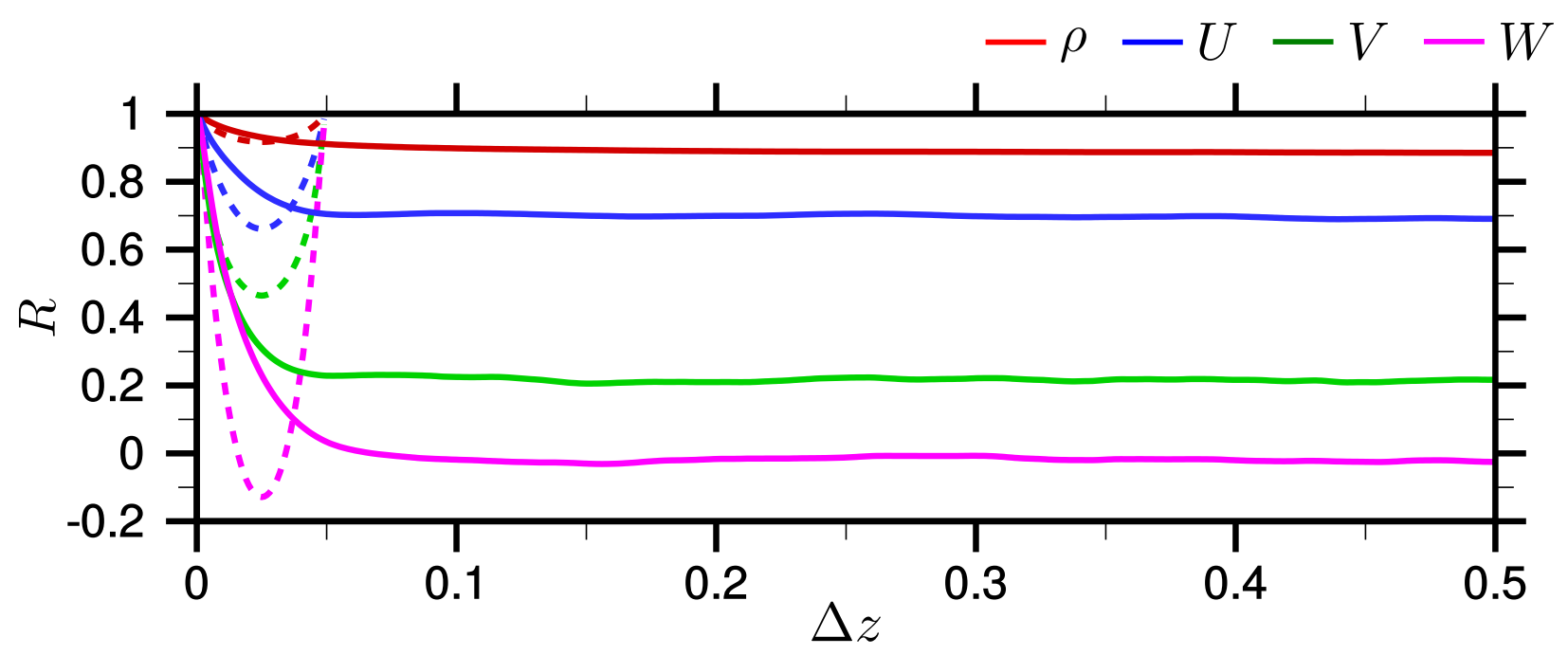

FIG. 7. Red, blue, green, and magenta lines show two-point correlations of density $(\rho)$, streamwise$(U)$, vertical- $(V)$, and spanwise-velocity $(W)$ components, respectively. Solid and dashed lines correspond to the wide- and narrow-domain case, respectively.

magenta dash-dotted curve after 210 time units) propagating at $M_{a} \approx-0.14$. Also neither the pressure ratio of $p_{2} / p_{1} \approx 1.07$ nor the absolute level of the peak pressure in between the compression and expansion, i.e. $p_{2} \approx 1.09$ vary significantly in that region. The green curves are interrupted just before half-chord at $x \approx 0.4$, where the flow near the shock foot does not turn subsonic at the monitored location. Parallel to the shock waves, there are compression waves (so-called "separation shocks") moving ahead with the same speed and forming a lambda-shock structure with the mean shock, which is typical for laminar shockwave/boundary-layer interactions [13, [57]. Both waves eventually disappear at $x \approx 0.2$, where the pressure ratio increases to $p_{2} / p_{1} \approx 1.11$, and then vanish shortly after a brief downstream excursion. It is interesting to note that the continuations of the black dotted lines corresponding to the shock speed always intersect the front sonic line at their most downstream position, even though no shock features are observed in that region, suggesting that the upstream wave-propagation effects continue even when the shock themselves are no longer visible. Further discussions of the unsteady flowfield will be provided in the next section, after we conclude the assessment of spanwise domain width effects.

A common method of assessing the suitability of the spanwise domain width is via twopoint correlations, which in homogeneous directions should drop to zero over half the domain 
width. Here, we consider a fixed position close to the suction-side trailing edge with a wallnormal distance of $\Delta y \approx 0.06(x=1.0035$ and $y=-0.0015)$. The averaged two-point correlation $R$ is calculated according to

$$
\begin{aligned}
& R_{(\Delta k)}=\frac{1}{N_{z} N_{t}} \sum_{k=1}^{N_{z}} \sum_{t=1}^{N_{t}} \frac{q_{(k, t)}^{\prime} q_{(k+\Delta k, t)}^{\prime}}{\left|q_{(k, t)}^{\prime}\right|\left|q_{(k+\Delta k, t)}^{\prime}\right|} \text { for } \Delta k \in\left[0, N_{z}\right], t \in\left[0, N_{t}\right] \\
& \Delta z=\frac{L_{z}}{N_{z}} \Delta k,
\end{aligned}
$$

where the vector $q_{(k,:)}^{\prime}$ contains a time series of the fluctuations in the quantity of interest at a fixed position in the $x y$-plane at the $k^{t h}$ point in the spanwise direction, which is projected onto vector $q_{(k+\Delta k,:)}^{\prime}$ and normalised by the magnitudes of both vectors. Finally, the average value is calculated for each $\Delta k$, looping through each point in the spanwise direction $(1<$ $k<N_{k} / 2$ ), and transformed to the physical distance $\Delta z$, using equation 18 . Figure 7 shows the two-point correlations for both cases (solid lines for the wide domain and dashed lines for the narrow domain), where the red, blue, green, and magenta lines correspond to density $(\rho)$, streamwise- $(U)$, vertical- $(V)$, and spanwise-velocity $(W)$ components, respectively. The extreme cases for the wide domain are the density, which remains highly correlated $(R=0.9)$, and the spanwise velocity, which drops to $R=0$ for $\Delta z>0.075$. What we are seeing in the two-point correlations is the effect of two distinct phenomena in the flowfield. The periodic motion associated with buffet (as shown in the next section) is essentially twodimensional, hence the density field remains highly correlated across the span. However, the superimposed turbulence is three-dimensional and contained within the domain, so that the spanwise velocity correlations drop to zero within $\Delta z=0.075$. The spanwise velocity is different to the other velocity components since it has a spanwise-mean value that is constant (equal to zero) during all phases of the buffet cycle.

In the narrow domain (shown with the dashed lines in figure 7 for the full range of possible $\Delta z)$ the spanwise two-point correlations do not decay to zero even for the spanwise velocity component. Despite this, and in common with experience of other flows in restricted (but not minimal) domains [58], the flow phenomena are representative of the wider domain case, so that such narrow-domain simulations can still be useful. 


\section{FURTHER ANALYSIS OF THE WIDE DOMAIN CASE}

In this section we look in detail at statistics and instantaneous flowfields from representative phases of the buffet cycle, exploiting the large amounts of data available for the wide domain case. To begin with, we focus in sub-section $\mathrm{VA}$ on phase-averaged data collected during high and low lift phases (denoted by HLP and LLP, respectively). In sub-section $\mathrm{VB}$ we analyse instantaneous 3D visualisations at representative time instances during high and low-lift phases, which allow us to study the spanwise characteristics of transition, shock waves, and low-frequency phenomena. Finally, in sub-section VC, we consider the global behaviour of low-frequency phenomena associated with transonic buffet, including the flow over the pressure side of the airfoil.

\section{A. Phase averaged flowfields}

The regularity of the buffet cycle in wide domain case allows us to extract reliable phaseaveraged statistics. Figure 8(a) shows in red and blue the sections of the lift history that were used to create averages over the high- and low-lift phases of the buffet cycle. Parts (b) and (c) of the figure show respectively the pressure drag and skin-friction drag coefficient, with the trailing-edge pressure measured at the upper-side corner in part (d). The pressure drag is broadly in phase with the lift coefficient, although the pressure drag has a slightly delayed peak and has a sharper drop during the decreasing-lift part of the cycle. Skin friction and trailing edge pressure are roughly in phase with each other (the trailing edge pressure lags slightly), and both lead the lift by about a quarter of a cycle. The friction drag, for example, reaches a minimum when transition takes place at its most downstream position $(x>0.7)$, just before the lift reaches its minimum.

Figure 9 shows in red and blue (a) wall-pressure and (b) skin-friction coefficient as a function of $x$ corresponding to high- and low-lift phases in figure 8(a). Part (a) of figure 9 is continued into the near wake to show the pressure variations downstream of the trailing edge. Key features are identified on the figure. Points H1 and L1 correspond to the first occurrences of flow separation $\left(C_{f}=0\right)$ on the upper surface (denoted with the solid lines). The initial separation is significantly earlier in the low-lift phase, after which there is an

extended region with $C_{f}$ close to zero. Although the separation is later in the high-lift 


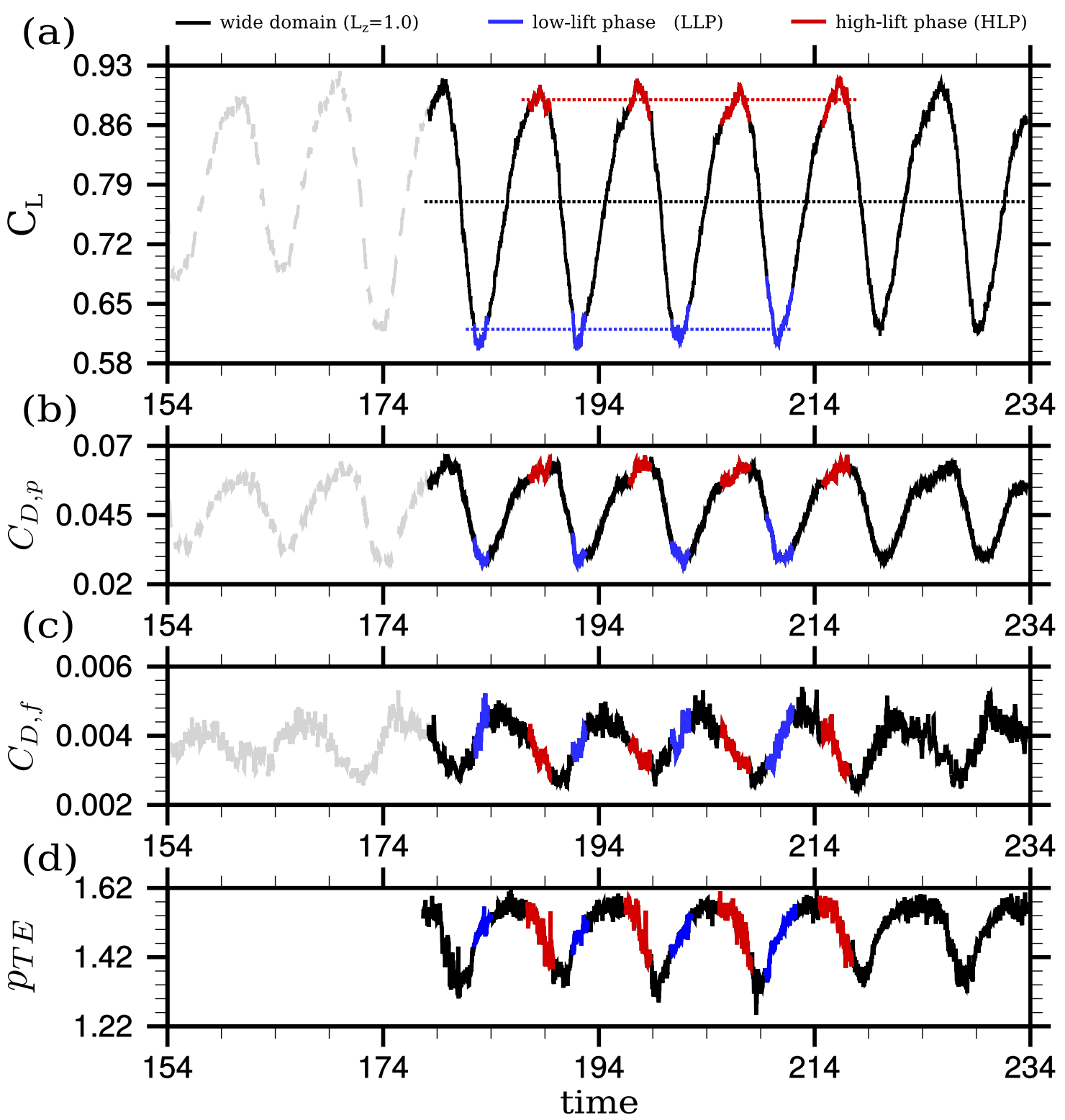

FIG. 8. Extraction of high-lift and low-lift statistics: (a) variation of lift coefficient with time, showing in red the high-lift samples and in blue the low-lift samples, (b) pressure-drag coefficient, (c) skin-friction drag coefficient, and (d) trailing-edge pressure.

phase, there is still an extended region of reverse flow present. Points H2 and L2 correspond to the crossing of the critical pressure, i.e. the isentropic sonic condition and it is notable that in both cases these points correspond to the minimum skin friction from 9 (b), i.e. with the location of the peak strength of the reverse-flow vortex that precedes reattachment. The pressure gradient at $\mathrm{H} 2$ and L2 and the upstream-pressure plateau hardly changes during the 


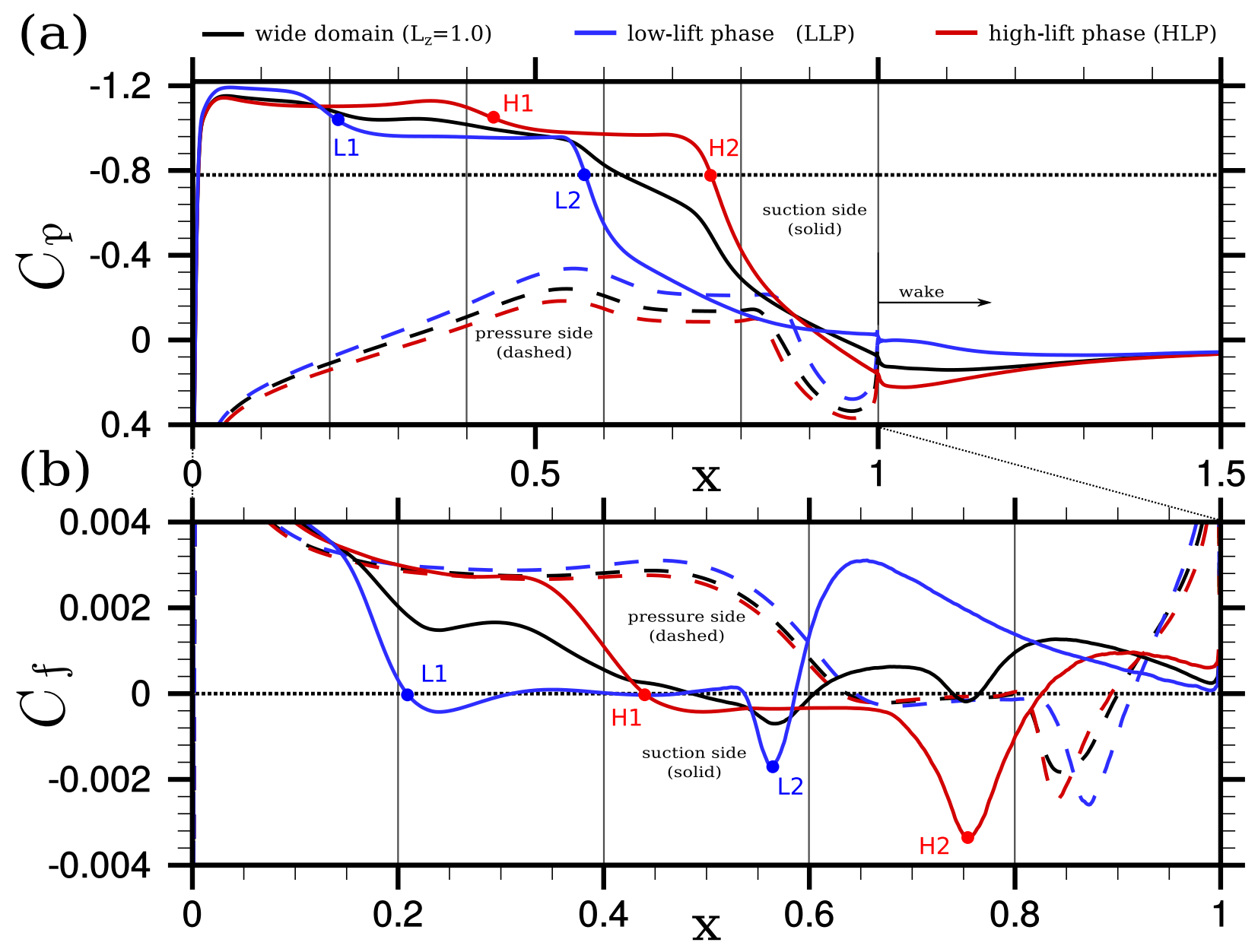

FIG. 9. Extraction of high-lift (red) and low-lift (blue) statistics corresponding to figure 8; (a) pressure coefficient, including the critical value denoted by the dash-dotted black line, and (b) skin friction coefficient. The upper surface is shown with solid lines and the lower surface with dashed lines.

low-frequency cycles, while the blue curve flattens at $x>0.6$ converging to higher trailingedge pressure. Considering the Rankine-Hugniot equations modelling the pressure jump across a shock wave, the shock strength is solely defined by the pressure and relative Mach number of the shock wave. This would suggest that shock waves terminating the supersonic region at high- and low-lift phases have the same strength on average. It is also interesting to note that, superficially, the $C_{p}$-curves in figure 9 (a) resemble the picture we would expect for a single shock wave oscillating back and forth over $0.5<x<0.8$. Strong gradients are removed by time averaging, so that the black curve reminds to full-time statistics of 
TABLE V. Aerodynamic coefficients.

\begin{tabular}{ccccccc}
\hline Name & $C_{L} / C_{D}$ & $C_{L}$ & $C_{D}$ & $C_{D, f}$ & $C_{D, p}$ & $\left.C_{D, p}\right|_{T E}$ \\
\hline HLP & $17.91(-12 \%)$ & $0.89(+16 \%)$ & $0.049(+29 \%)$ & 0.0036 & 0.046 & -0.014 \\
total $^{*}$ & $20.28( \pm 0 \%)$ & $0.77( \pm 0 \%)$ & $0.038( \pm 0 \%)$ & 0.0038 & 0.034 & -0.014 \\
LLP & $29.24(+44 \%)$ & $0.62(-19 \%)$ & $0.021(-45 \%)$ & 0.0039 & 0.017 & -0.013 \\
\hline
\end{tabular}

values in bracket denote the percental deviation from total*

experiments at high Reynolds numbers [31]. However, the instantaneous flowfield looks quite different, as we observe multiple upstream propagating shock waves in figure 6(b) and animations (https://youtu.be/pjRzeSYb4AY). The animation [59] shows contours of axial pressure gradient similar to figure 3(a). Due to the decreased resolution above the fore part of the airfoil $(x<0.2)$, grid-to-grid point oscillations can occasionally be seen as multiple stripes parallel to oblique shock waves in the visualisations, e.g. at $t=184$. These grid-togrid point oscillations are exaggerated by the narrow colour-map scaling $(-5<\partial p / \partial x<5)$ and show no impact on the boundary layer.

Table $\mathrm{V}$ summarises the aerodynamic coefficients from these two flow phases and gives their relative departure from the time average, which is up to $19 \%$ in the case of the lift coefficient and $45 \%$ for the drag coefficient. However, it is also interesting that some quantities, such as the friction contribution to drag and the base-drag coefficient $\left.C_{D, p}\right|_{T E}$ (integrating pressure contributions along the blunt trailing edge) vary by less than $10 \%$.

To link more clearly the boundary-layer behaviour with the external flow field, figure 10 shows span-averaged contours of long-time averaged Mach number, comparing the corresponding sonic line in black with the same line in the high- and low-lift phases shown by the red and blue lines respectively. Part (a) of the figure shows the whole supersonic region, while part (b) focuses on the boundary layer. Both parts of the figure are distorted by stretching the $y$ co-ordinate, more severely in (b). As well as the sonic line, the figures show the $u=0$ contour (in magenta and light-blue for low and high lift phases respectively) and dashed lines show the region of strongest adverse streamwise pressure gradient. While the region of flow reversal on the pressure side hardly changes between the high and low-lift phases, we can observe the significantly larger region of flow reversal consistent with the skin 


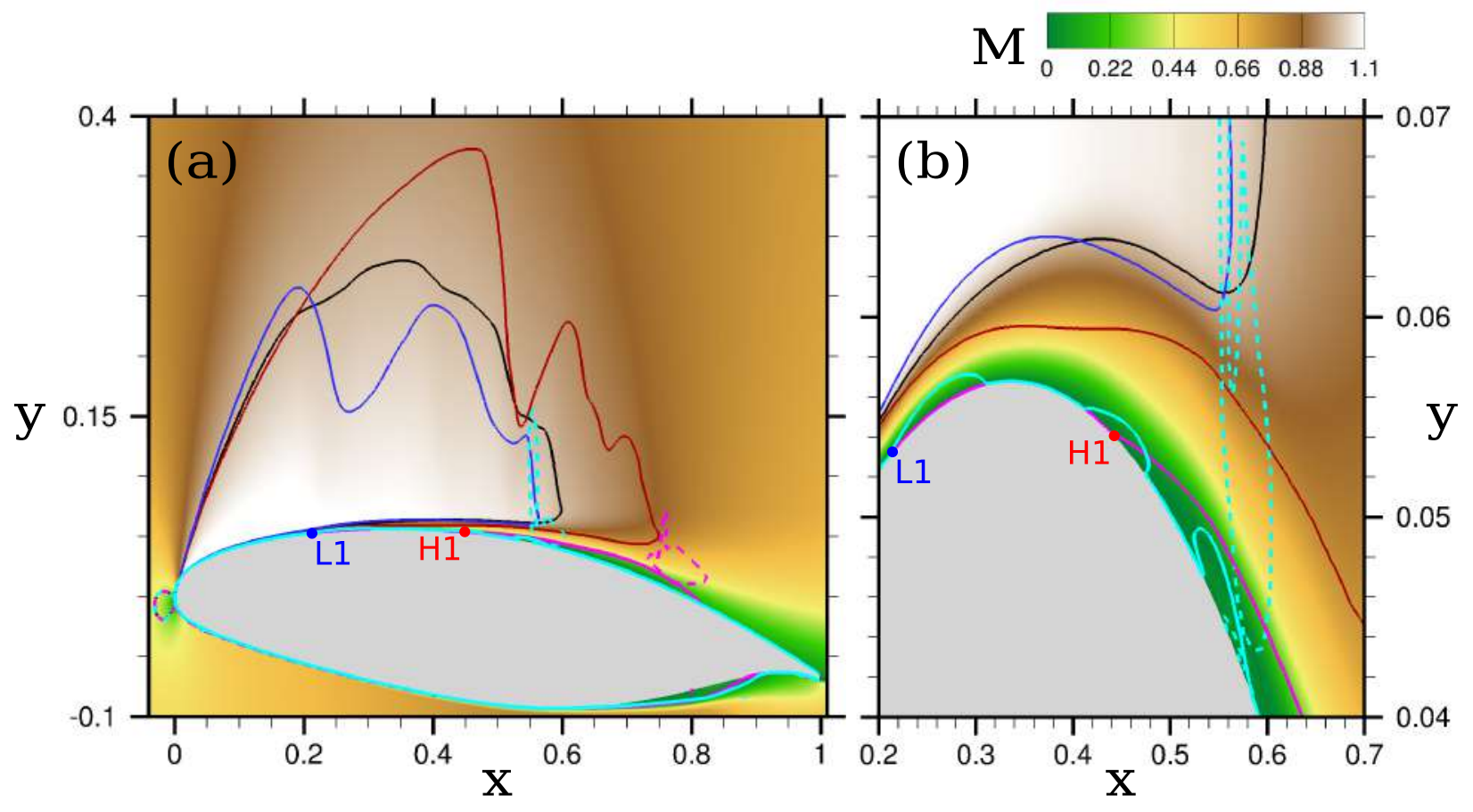

FIG. 10. Contours showing the time- and span-averaged Mach number of case $C 0$. Solid black, red, and blue curves denote sonic lines corresponding to full time-averaged, high-lift, and low-lift phase-averaged flowfields, respectively. Dashed light-blue and magenta lines indicate high adverse pressure gradient for low-lift phase (LLP) and high-lift phase (HLP), respectively. Magenta and light-blue solid lines denote iso-curves of streamwise velocity $u=0$. Both figures (a) and (b) are distorted.

friction plots on figure 9(c). The supersonic region is clearly larger in the high-lift phase, with oscillations in the sonic line after the half chord showing the time-averaged effect of the shock/expansion combination, while such oscillations extend throughout the supersonic region in the low-lift phase. As seen in the $x / t$ diagrams there are more than one of these shock/expansion waves, in contrast to the standard picture of buffet involving a single shock oscillating back and forth. The region of highest pressure gradient (the dashed lines) can be interpreted at the averaged effect of the multiple propagating shock waves seen in the present configuration and is, again, more consistent with the standard picture of where a shock would occur: further upstream during the low lift phases and further downstream during the high-lift phase. In addition to longitudinal regions oriented orthogonal to streamlines, which are reminiscent of shock waves, we also observe regions near the wall showing 
high pressure gradients. These regions seem to be caused by upstream propagating acoustic waves accumulating near the sonic line. These regions are larger during high-lift phases and may be the reason for the increased pressure gradient at the rear part of the airfoil (observed in figure $9(b))$.

\section{B. Instantaneous $3 \mathrm{D}$ visualisations}

More information, particularly relating to the transition process, can be gained from visualisations of the flowfields at high- and low-lift instants. These were identified on figure 4 as time instants WH and WL respectively for the wide domain case. A common practice to visualise vortex structures is the so-called Q-criterion, defining vortices as a local imbalance of vorticity and shear strain according to

$$
Q=\frac{1}{2}\left(\|\Omega\|^{2}-\|S\|^{2}\right)=\frac{1}{2}\left(u_{i i} u_{j j}-u_{i j} u_{j i}\right),
$$

where $\Omega$ denotes the vorticity tensor and $S$ the strain-rate tensor. Figure 11 shows a combination of side $(x-y)$ and top views $(x-z)$ using an isosurface of $Q=100$ colored by the magnitude of the velocity, which serves to identify the key flow features for further discussion. The Q-criteria surfaces are only plotted for the upper part of the wake to help distinguish coherent vortical structures. In addition, the side view shows contours of the streamwise pressure gradient and the white line is the instantaneous sonic line. At the high lift condition the separation point is further aft and we have a much narrower wake compared to the low lift condition. Associated with this we have a large supersonic region, extending beyond the half-chord location in the high lift condition, whereas in the low-lift condition

we have a series of shocks and expansions over the front part of the airfoil, reminiscent of the shock-train of [60].

In the top views of figure 11 we can observe the transition process and get an impression of the size of turbulent structures in the separated flow region and extending into the wake. In the low-lift phase we see clear evidence for Kelvin-Helmholtz roll-ups (i.e. arising from an inflectional instability) of the separated shear layer, with about 6 successive roll-ups visible for $x<0.5$. Similar structures were observed over a high-pressure turbine vane cascade at a comparable Reynolds number and predicted by linear stability methods in boundary layers near flow separation (velocity profiles exhibit deflection points) [61. The presence of Kelvin- 

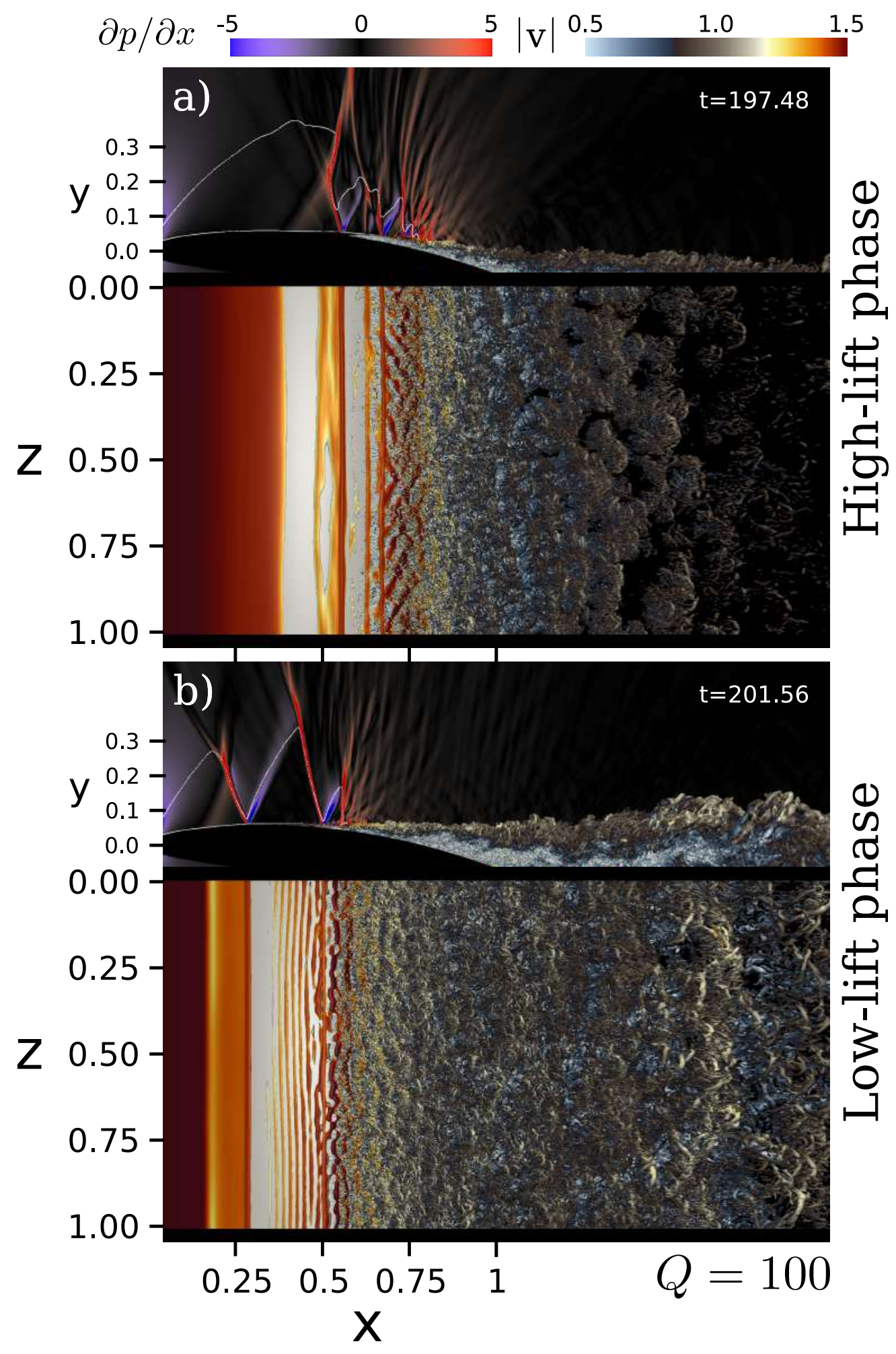

FIG. 11. Instantaneous 2D slices (side-view $(x-y)$ and top-down view $(x-z)$ ) showing Qcriterion surfaces $(Q=100)$ coloured by velocity magnitude at (a) high-lift and (b) low-lift phase corresponding to labels WH and WL in figure 4, respectively. 
Helmholtz roll ups does not exclude a possible connection to Tollmien-Schlichting waves in the upstream boundary layer, though the significant amplification seen here is associated with the strong inflectional instability.

These $Q$-structures are continuous across the span, with a slight waviness, which may be caused by linear instabilities with large spanwise wave numbers [61]. The first spanwise dislocations are visible at the mid-chord location $x=0.5$ followed by a rapid breakdown to turbulence, attributed in previous work [37] to a three-dimensional self-sustained instability of the unsteady large-scale structures. In the developing flow downstream of the transition we observe the formation of larger scale structures, but these are still comfortably contained within the large computational domain.

Additional features of the flow upstream of transition are $Q$-structures that form as the flow is turned at the edge of the boundary layer due to shock/expansion combinations that cause a local thickening and then thinning of the boundary layer. Examples are seen at $x \approx 0.28$ in the high-lift phase and $x=0.55$ in the low-lift phase in figure 11(a). We have observed similar structures in shock-induced separation bubbles, where the flow at the apex of the bubble turns rapidly in response to the impinging shock wave, also resulting in a reflected expansion wave. The interesting feature here is that these structures show almost no variation across the span, even in the wide domain, despite the turbulent flow regions being fully three-dimensional.

In the high-lift phase, seen on figure 11(a), transition appears to follow directly from the $Q$-structures that form from the shock-wave/boundary-layer interaction [62]. The spanwise structure at $x=0.66$ is followed by a cross-hatched pattern of structures corresponding to a spanwise wave-length of $\lambda_{z} \approx 0.07$ and suggesting an oblique mode of instability preceding the final breakdown to turbulence 63, 64]. The transition process continues up to $x=0.8$ in this case, after which larger-scale turbulent features emerge. The wake is more intermittent in the high-lift phase and shows large-scale structures roughly up to a fifth of the domain width.

Figure 12 shows the variation of the Q-structures during the complete buffet cycle, considering four intermediate phases (in parts b, c, e and f) in between the high-lift phase in part (a) and the low-lift phase in part (d). The high and low-lift phases are also shown respectively in perspective (stereo $3 \mathrm{D}$ ) views in the top right and bottom left of the figure, which show more clearly the large-scale structures in the near-wake region. The main 


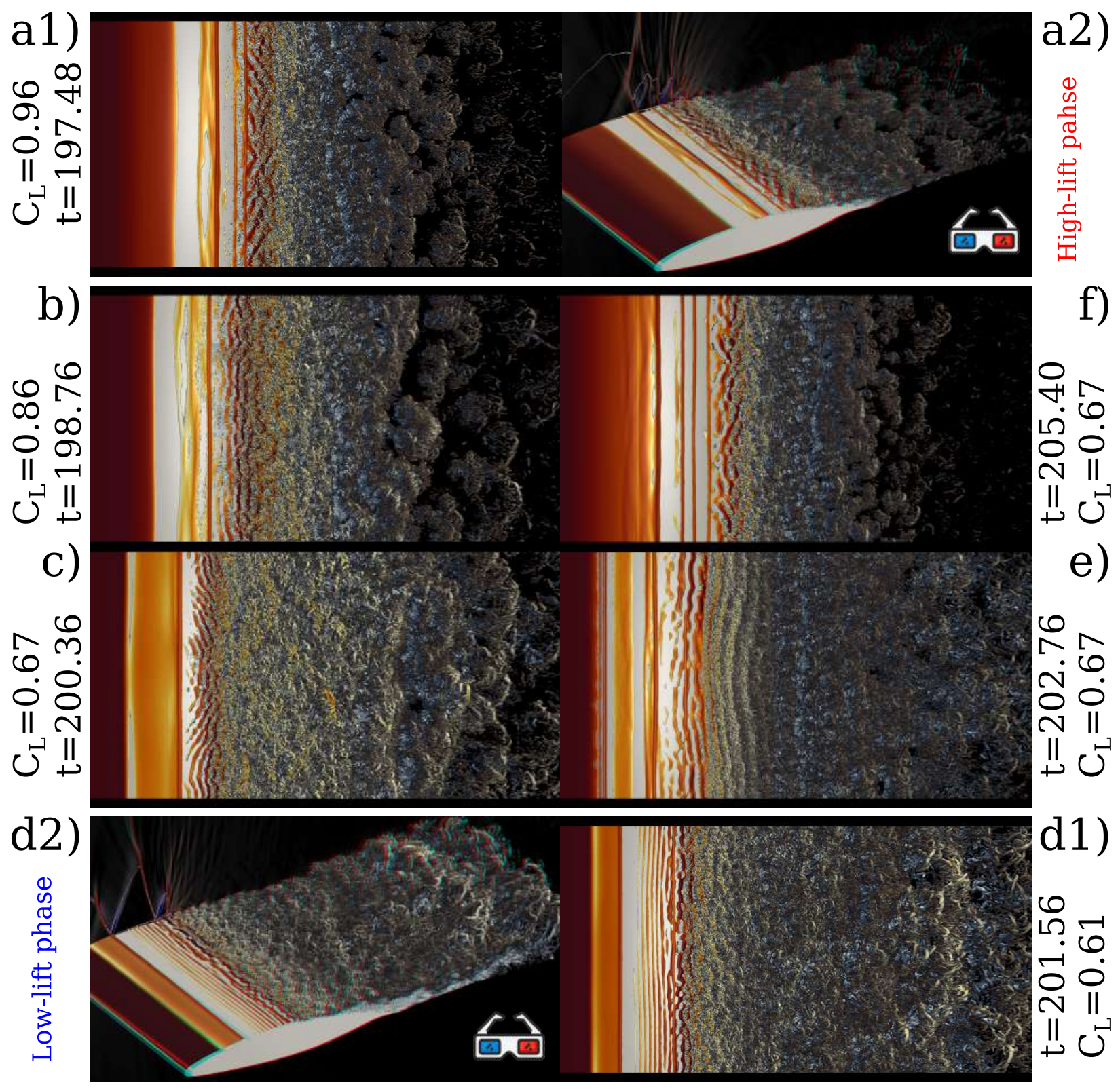

FIG. 12. Top-down view $(x-z))$ showing $Q$-criterion surfaces $(Q=100)$ coloured by velocity magnitude. Perspective (stereo 3D) views at (a2) HLP and (d2) LLP.

features of the other plots have already been described in connection with figure 11. Considering the whole buffet cycle in figure 12 we see the forward and backward movement of the separation location and the strict two-dimensionality of the shock-train patterns. The only indication of potentially larger-scale structures is the spanwise waviness of the transitional flow structures seen in figure 12(e), but these are a weak feature and are only present for a small part of the buffet cycle. Similar to the present study, no significant 3D structures 
were observed in experiments by [65] on a two-dimensional NASA CRM airfoil, however stall and buffet cells have been reported by [66, 67] at spanwise whavelengths $\lambda_{z}>1 c$ for different airfoil geometries. One would have expected to see significant larger scale features already in the present wide-domain simulation in the case that stall/buffet-cell behaviour was relevant for this airfoil at this flow condition, although it is not possible to say for sure that they would not form in even wider computational domains.

\section{Global behaviour of low-frequency phenomena}

Having confirmed that the low-frequency phenomenon associated with buffet has a significant $2 \mathrm{D}$ component, we next consider additional $x / t$ diagrams at $z=0$. While we focused in figure 6 on the behaviour of shock waves and acoustic waves, we now consider the overall characteristics of the flow undergoing buffet. Contours in figure 13 show fluctuations of (a) isentropic Mach number fluctuations at the airfoil surface and (b) Mach number fluctuations along a monitor line outside the boundary layer (at $\Delta y=0.05$ from the surface). The left part of both plots in figure 13 show the pressure sides, while the right part shows the suction sides (the sketches on the top indicate active monitor curves by solid lines and inactive ones by dotted curves). The $x$-axis of the pressure-side plots has been reversed so that the trailing edge corresponds to the extreme left and right edges of the figure, while the leading edge is located at the centre. The isentropic Mach number is commonly used to estimate locations of shock waves in experiments, using only the wall-pressure [68] and is computed here according to

$$
M_{i s}=\sqrt{\left(\left(\frac{p_{0}}{p}\right)^{\frac{\gamma-1}{\gamma}}-1\right) \frac{2}{\gamma-1}} .
$$

Fluctuations of Mach numbers in figure 13 are defined as

$$
M^{\prime}=\frac{M-\bar{M}}{\bar{M}},
$$

where $\bar{M}$ denotes the local time average. While figure 6 focused our attention on shock waves and acoustic phenomena associated with intermediate and small time scales $(S t>0.4)$, figure 13 highlights low-frequency phenomena corresponding to $S t<0.4$.

Considering firstly figure 13(a) at the airfoil surface, we observe at the right of the figure a periodic pattern moving downstream with a speed of $v_{c} \approx 0.098$, representing a slow 


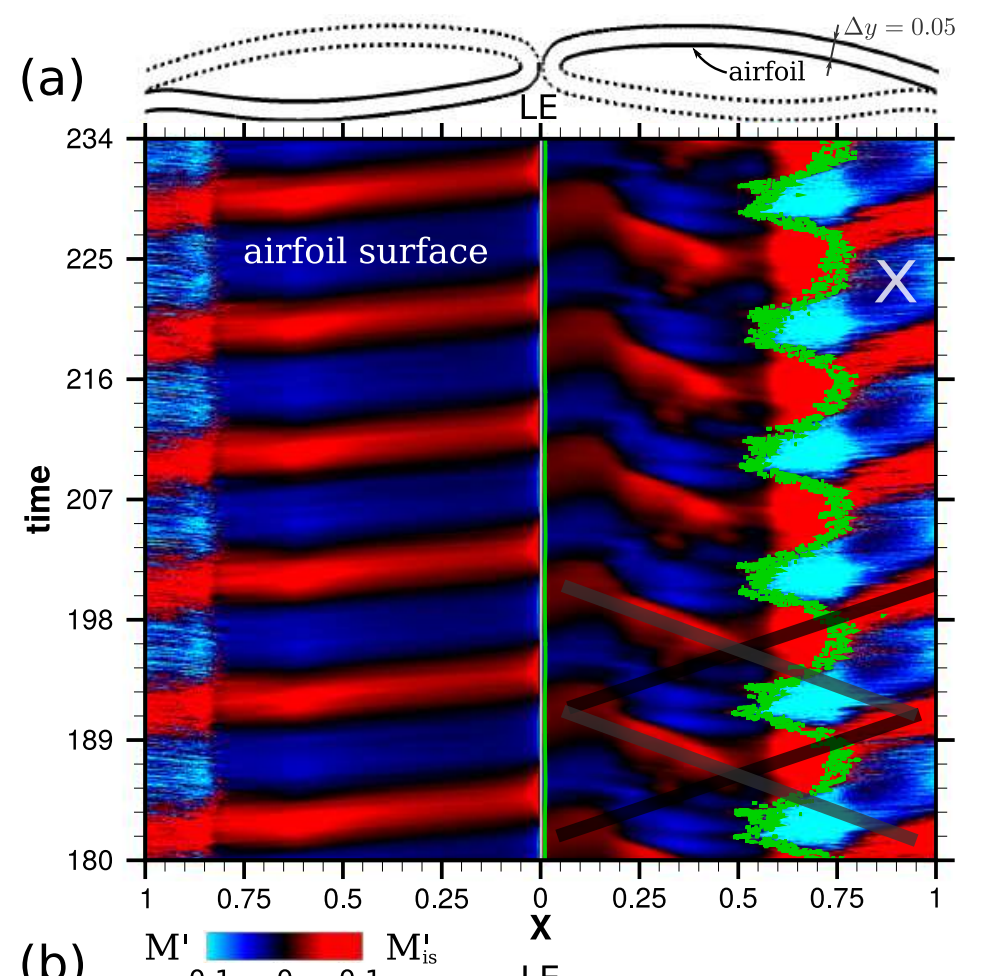

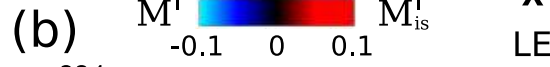

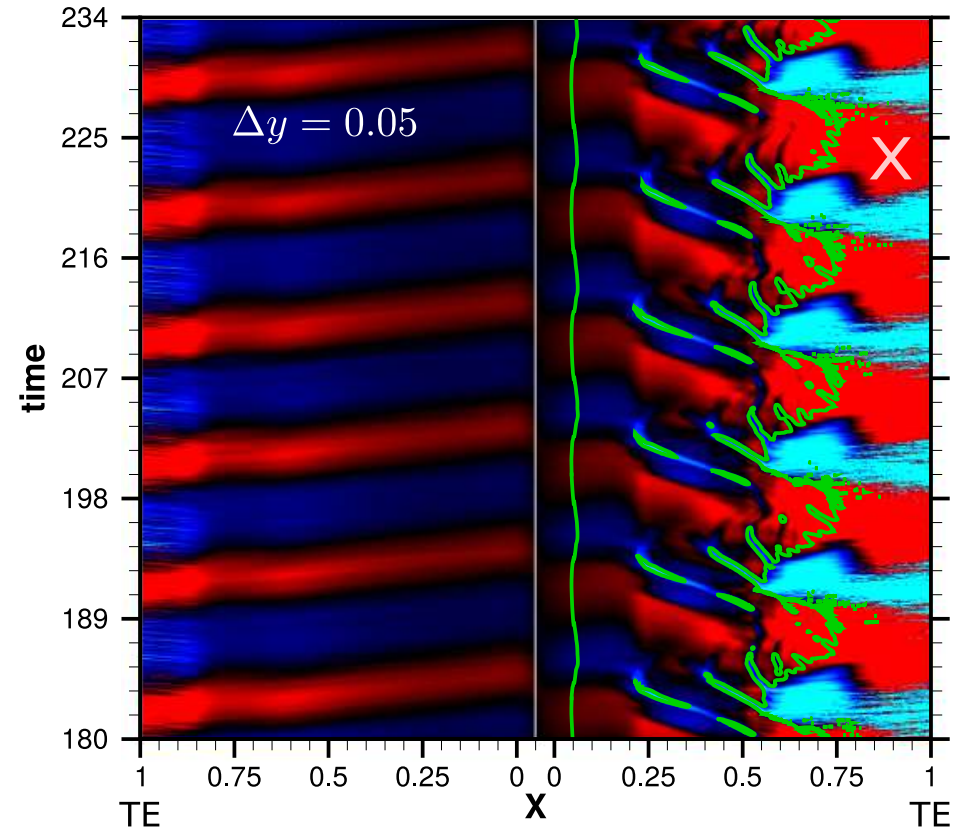

FIG. 13. Contours showing (a) isentropic Mach number fluctuations at the airfoil surface and (b) Mach number fluctuations along an isocurve outside the boundary layer as a function of space and time, respectively. The green lines denote sonic lines. 
adjustment of the reattached boundary layer. After encountering the trailing edge, this pattern then continues propagating along the pressure side, but in the upstream direction at a velocity of $v_{u}=-0.364$ (deduced from the positive slope on the left plot in figure 13(a)). At the leading edge, we observe a phase shift between suction and pressure side, which means that the flow is accelerating on one side, while it is decelerating on the other side. On the upper side we can observe weak indications of downstream-moving structures in the Mach contours near the leading edge (positive slope on the right plot in figure 13(a) corresponding to a velocity of $v_{c} \approx 0.113$ at $x \approx 0.2$ ). For $x>0.2$, these structures then disappear or are hidden by upstream-propagating structures with a negative slope on the right plot in figure 13 (a) in the range of $0.2<x<0.6$. Near the transition region we observe a patchy green sonic line undergoing the low-frequency oscillations associated with transonic buffet. This is reminiscent to the traditional picture of transonic buffet, where only a single shock wave is moving back and forth [27] 69].

The shock waves are clearer in figure 13(b), showing fluctuations of Mach number along a monitor line $\Delta y=0.05$ away from the surface. The green sonic lines correspond to those shown previously in figure 6(b). The upstream-propagating shock waves seem to move only during phases where Mach numbers are lower than the time average corresponding to blue corridors, in good agreement with the discussion of part (a) of the figure. While we observe multiple shock waves per low-frequency cycle at $x \approx 0.5$, we observe only one shock wave per cycle at positions further upstream $(x<0.3)$. A striking qualitative difference between the two monitor curves shown in figures 13(a) and (b) is observed near the suction-side trailing edge, where the structures within and outside the boundary layer are completely different, as for example highlighted at $t \approx 225$ by the white cross. The underlying wave structure is revealed in the isentropic Mach number fluctuations, but obscured in the Mach number plot. Remaining features of figure 13(b) agree well with (a) or have been already discussed in connection with the pressure-gradient field in figure 6(b).

Lines have been added to the first two low-frequency cycles of the upper side plot in figure $13(\mathrm{a})$, to illustrate the form of a standing wave pattern between suction-side leading edge and trailing edge, as previously seen in [37]. The role of the trailing edge in the buffet cycle has been the subject of some previous experiments, for example by adding splitter plates to the trailing edge [70]. Even though buffet could be suppressed in some cases, there were also cases showing no effect or just a change in the buffet characteristics. This suggests 
that the behaviour on the pressure side can affect the low-frequency phenomenon, but is not necessarily part of the mechanism that drives the buffet. The work of [71] supports this assumption, as they applied selective frequency filtering on the pressure side without affecting the buffet phenomenon significantly. This would mean that, also in our case, the pressure side is only reacting to changes in the flow field induced by the suction side via the trailing edge.

A question arising from the present work is the significance of the leading-edge phase shift. This could just be a random shift, due to the different arrival times of waves from the lower and upper sides, or it could be that the weak downstream-propagating wave, observed on the suction side near the leading edge, plays a role in connecting the pressure side disturbances to the boundary layer on the suction side, upstream of its separation point. This aspect deserves further study.

\section{CONCLUSIONS}

A comparison has been made of transonic buffet, captured in simulation with widely different spanwise domain sizes and span-periodic conditions. The configuration is based on an unswept laminar-flow supercritical airfoil (Dassault Aviation's V2C profile) at free stream Mach number of 0.7 and Reynolds number of 500,000. The numerical approach was based on large-eddy simulation, using a spectral estimate to control the level of local filtering applied. Initially a narrow-domain simulation was used to validate the numerical approach against a direct numerical simulation. Then an LES was run in a domain twenty times wider, with the same $x-y$ grid and twenty times the number of points in the spanwise direction.

It was found that the narrow domain was sufficient to capture the flow physics of the buffet cycle, with lift to drag ratio for example being within $3 \%$. In terms of usual measures used to assess the effect of domain width it was found that two-point correlations mostly failed to fall to zero. This is due to the continued two-dimensionality of the potential flow outside the boundary layer, including the pattern of upstream-propagating shock waves. The exception was the spanwise velocity which, since it only depends on the turbulent flow, fell to zero within a spanwise separation an order of magnitude smaller than the domain width. 
Quantitative effects of the increasing domain width included the increasing regularity of the buffet cycle and the increased amplitude. These effects were both attributed to the increased capture of large-scale structures near the trailing edge. The increased energy of these can lead to high buffet amplitudes and the larger statistical sample of such structures in the wide domain can lead to greater regularity, since the conversion to two-dimensional waves at the trailing edge is averaged over more structures.

The transition mechanism was studied at representative instants of high and low-lift phases of the buffet cycle. At low-lift the transition proceeded via Kelvin-Helmholtz structures and a rapid breakdown consistent of a kind seen before. At the high-lift condition the transition proceeds from span-uniform structures imposed by upstream-propagating shock waves reflecting as expansion waves. Such structures show up clearly in $Q$-isosurfaces, with transition following via oblique modes rather than via two-dimensional Kelvin-Helmholtz waves. There was no sign of stall cells, with the only large-scale three-dimensionality being a slight waviness in the Kelvin-Helmholtz structures.

Phase-averaged statistics from the wide-domain simulation provide an impression of a single back and forth moving shock wave, while instantaneous data show multiple upstreampropagating shock waves. Considering the global flow field, the pressure side was found to be linked with the unsteady flow over the suction side via upstream propagation of acoustic waves originating at the trailing edge that can be traced back to the separation region by a slow downstream propagation that is visible in isentropic Mach number contours. Upstreampropagating waves also appear over the suction side where they may connect to the pressure side waves near the leading edge, the precise role of which needs further study.

\section{OPEN AVAILABILITY OF PERTINENT DATA}

The source code to compile the SE-ILES grid (it was not possible to include Dassault Aviation's V2C profile), a python code for two-point correlation, data corresponding to figures $2,3,4,5,6,8,9,13$, and animations are openly available from the University of Southampton repository (https://doi.org/10.5258/SOTON/D1484). Animations are also accessible via youtube [72].

The corresponding data-set also contains additional quantities along monitor lines at the airfoil surface and outside the boundary layer as a function of chord position and time, which 
were not discussed in this work, but may be of interest of the research community.

\section{ACKNOWLEDGEMENT}

We are grateful for computational resources provided by the Partnership for Advanced Computing in Europe (PRACE grant entitled "TRAAD - TRansonic Airfoil AeroDynamics") on the HazelHen super-computer (HLRS), by the Engineering and Physical Sciences Research Council (EPSRC), ARCHER (Leadership grant entitled "Transonic flow over an aerofoil"), and UKTC (EPSRC grant EP/L000261/1) on the UK national super-computer ARCHER, and by the University of Southampton on the Iridis clusters. MZ was supported by an EPSRC studentship award (reference 1665277) and by an EPSRC grant entitled "Extending the buffet envelope: step change in data quantity and quality of analysis" (EP/R037027/1).

[1] D. G. Mabey, A Review of Scale Effects in Unsteady Aerodynamics, in ICAS proceedings (1990).

[2] E. Stanewsky and D. Basler, Aircraft dynamic loads due to flow separation. Sorrento, Italy: AGARD CP-483 (1990) pp. 4:1-11.

[3] B. H. K. Lee, H. Murty, and H. Jiang, Role of Kutta waves on oscillatory shock motion on an airfoil, AIAA Journal 32, 789 (1994).

[4] N. F. Giannelis, G. A. Vio, and O. Levinski, A review of recent developments in the understanding of transonic shock buffet, Progress in Aerospace Sciences 92, 39 (2017).

[5] H. Tijdeman, On the Motion of Shock Waves on an Airfoil with Oscillating Flap, Symposium Transsonicum II. International Union of Theoretical and Applied Mechanics (1975).

[6] M. T. Landahl, Some Developments in Unsteady Transonic Flow Research, in Symposium Transsonicum II (1977) pp. 1-32.

[7] S. Raghunathan, M. Gillan, R. Cooper, R. Mitchell, and J. Cole, Shock oscillations on biconvex aerofoils, Aerospace Science and Technology 3, 1 (1999)

[8] B. Lee, Self-sustained shock oscillations on airfoils at transonic speeds, Progress in Aerospace Sciences 37, 147 (2001), 
[9] C. Tulita, E. Benard, and S. Raghunathan, Transonic Periodic Flow Subject to Adaptive Bump, in 41st Aerospace Sciences Meeting and Exhibit, January (American Institute of Aeronautics and Astronautics, Reston, Virigina, 2003) pp. 1-15.

[10] F. Tamaki, Experimental Studies on the Stability of the Transonic Flow past Airfoils, Journal of the Physical Society of Japan 12, 544 (1957).

[11] L. Trilling, Oscillating Shock Boundary-Layer Interaction, Journal of the Aerospace Sciences 25, 301 (1958).

[12] R. J. Hakkinen, I. Greber, L. Trilling, and S. Abarbanel, The Interaction of an Oblique Shock Wave with a Laminar Boundary Layer, Tech. Rep. (Massachusetts Inst. of Tech., Cambridge, MA, United States, 1959).

[13] K. Karashima, Instability of Shock Wave on Thin Airfoil in High Subsonic Flow, Tech. Rep. (Aeronautical Research Institute, University of Tokyo, 1961).

[14] R. Dvorak, On the unsteady boundary layer-shock wave interaction in the lower transonic region., in Fluid Dynamics Transactions (Elsevier, 1965) pp. 63-74.

[15] E. Garnier and S. Deck, Large-eddy simulation of transonic buffet over a supercritical airfoil, in Notes on Numerical Fluid Mechanics and Multidisciplinary Design, Vol. 110 (2010) pp. $135-141$.

[16] T. Kouchi, S. Yamaguchi, S. Koike, T. Nakajima, M. Sato, H. Kanda, and S. Yanase, Wavelet analysis of transonic buffet on a two-dimensional airfoil with vortex generators, Experiments in Fluids 57, 1 (2016).

[17] Y. Fukushima and S. Kawai, Wall-modeled Large-Eddy Simulation of Transonic Buffet over a Supercritical Airfoil at high Reynolds Number, in 55th AIAA Aerospace Sciences Meeting, January (American Institute of Aeronautics and Astronautics, Reston, Virginia, 2017) pp. $1-10$.

[18] J. D. Crouch, A. Garbaruk, and D. Magidov, Predicting the onset of flow unsteadiness based on global instability, Journal of Computational Physics 224, 924 (2007).

[19] F. Sartor, C. Mettot, and D. Sipp, Stability, Receptivity, and Sensitivity Analyses of Buffeting Transonic Flow over a Profile, AIAA Journal 53, 1980 (2015).

[20] S. Timme, Global instability of wing shock buffet, , 1 (2018), arXiv:1806.07299.

[21] V. Brunet, Advances in Hybrid RANS-LES Modelling, edited by S.-H. Peng and W. Haase, Notes on Numerical Fluid Mechanics and Multidisciplinary Design, Vol. 97 (Springer Berlin 
Heidelberg, Berlin, Heidelberg, 2008).

[22] M. Iovnovich and D. E. Raveh, Numerical Study of Shock Buffet on Three-Dimensional Wings, AIAA Journal 53, 449 (2015).

[23] F. Sartor and S. Timme, Mach number effects on buffeting flow on a half wing-body configuration, International Journal of Numerical Methods for Heat \& Fluid Flow 26, 2066 (2016).

[24] L. Masini, S. Timme, and A. J. Peace, Analysis of a civil aircraft wing transonic shock buffet experiment, Journal of Fluid Mechanics 884, A1 (2020).

[25] E. Paladini, S. Beneddine, J. Dandois, D. Sipp, and J.-C. Robinet, Transonic buffet instability: From two-dimensional airfoils to three-dimensional swept wings, Physical Review Fluids 4, 103906 (2019).

[26] J. B. Dor, A. Mignosi, A. Seraudie, and B. Benoit, Wind Tunnel Studies of Natural Shock Wave - Separation Instabilities for Transonic Airfoil Tests, in Symposium Transsonicum III (1989) pp. 417-427.

[27] D. G. Mabey, Beyond the buffet boundary, The Aeronautical Journal 10.1017/S0001924000040811 (1973).

[28] R. Bur, V. Brion, and P. Molton, An overview of recent experimental studies conducted in ONERA S3CH transonic wind tunnel, 29th Congress of the International Council of the Aeronautical Sciences, ICAS 2014 10.13140/2.1.1791.8407 (2014).

[29] J. Dandois, Experimental study of transonic buffet phenomenon on a 3D swept wing, Physics of Fluids 28, 016101 (2016).

[30] V. Brion, J. Dandois, R. Mayer, P. Reijasse, T. Lutz, and L. Jacquin, Laminar buffet and flow control, Proceedings of the Institution of Mechanical Engineers, Part G: Journal of Aerospace Engineering , 095441001882451 (2019).

[31] R. Placek and P. Ruchała, The flow separation development analysis in subsonic and transonic flow regime of the laminar airfoil, Transportation Research Procedia 29, 10.1016/j.trpro.2018.02.029 (2018).

[32] T. Davidson, Effect of incoming boundary layer state on flow development downstream of normal shock wave - boundary layer interactions, Phd thesis, University of Cambridge (2016).

[33] F. Grossi, Physics and modeling of unsteady shock wave/boundary layer interactions over transonic airfoils by numerical simulation, Ph.D. thesis, Institut National Polytechnique de Toulouse (2014). 
[34] D. Szubert, I. Asproulias, F. Grossi, R. Duvigneau, Y. Hoarau, and M. Braza, Numerical study of the turbulent transonic interaction and transition location effect involving optimisation around a supercritical airfoil., European Journal of Mechanics - B/Fluids 55, 10.5604/12314005.1213755 (2016).

[35] J. Sznajder and T. Kwiatkowski, Analysis of effects of shape and location of micro-turbulators on unsteady shockwave-boundary layer interactions in transonic flow, Journal of KONES Powertrain and Transport 23, 373 (2016).

[36] A. Memmolo, M. Bernardini, and S. Pirozzoli, Scrutiny of buffet mechanisms in transonic flow, International Journal of Numerical Methods for Heat \& Fluid Flow , 00 (2016).

[37] M. Zauner, N. De Tullio, and N. D. Sandham, Direct numerical simulations of transonic flow around an airfoil at moderate Reynolds numbers., AIAA Journal 57, 597 (2019).

[38] M. Zauner and N. D. Sandham, Modal Analysis of a Laminar-Flow Airfoil under Buffet Conditions at $\operatorname{Re}=500,000$, Flow, Turbulence and Combustion 10.1007/s10494-019-00087-z (2019).

[39] R. Vinuesa, S. M. Hosseini, A. Hanifi, D. S. Henningson, and P. Schlatter, Pressure-Gradient Turbulent Boundary Layers Developing Around a Wing Section, Flow, Turbulence and Combustion 99, 613 (2017).

[40] S. Deck, Numerical simulation of transonic buffet over an airfoil, AIAA journal 43, 1556 $(2005)$.

[41] J. Dandois, I. Mary, and V. Brion, Large-eddy simulation of laminar transonic buffet, Journal of Fluid Mechanics 850, 156 (2018),

[42] M. Zauner and N. D. Sandham, LES study of the three-dimensional behaviour of unswept wing sections at buffet conditions, in Direct and Large-Eddy Simulation XII (2019).

[43] N. A. Adams and S. Hickel, Implicit Large-Eddy Simulation: Theory and Application (Springer Berlin Heidelberg, Berlin, Heidelberg, 2009) pp. 743-750.

[44] A. Aspden, N. Nikiforakis, S. Dalziel, and J. Bell, Analysis of implicit LES methods, Communications in Applied Mathematics and Computational Science 3, 103 (2008).

[45] A. Sansica, Stability and Unsteadiness of Transitional Shock-Wave/Boundary-Layer Interactions in Supersonic Flows, PhD Thesis, University of Southampton , 179 (2015).

[46] N. De Tullio and N. D. Sandham, Direct numerical simulations and modal analysis of subsonic flow over swept airfoil sections, (2019), arXiv:1901.04727. 
[47] L. Jones, Numerical studies of the flow around an airfoil at low Reynolds number, Ph.D. thesis (2008).

[48] M. H. Carpenter, J. Nordström, and D. Gottlieb, A Stable and Conservative Interface Treatment of Arbitrary Spatial Accuracy, Journal of Computational Physics 148, 341 (1999).

[49] R. D. Sandberg and N. D. Sandham, Nonreflecting Zonal Characteristic Boundary Condition for Direct Numerical Simulation of Aerodynamic Sound, AIAA Journal 44, 402 (2006).

[50] H. Sandhu and N. Sandham, Report QMW-EP-1100, Faculty of Engineering, Queen Mary and West-field College, University of London, Tech. Rep. (Faculty of Engineering, Queen Mary and Westfield College, University of London, 1994).

[51] M. R. Visbal and D. V. Gaitonde, On the Use of Higher-Order Finite-Difference Schemes on Curvilinear and Deforming Meshes, Journal of Computational Physics 181, 155 (2002).

[52] D. V. Gaitonde, J. S. Shang, and J. L. Young, Practical aspects of higher-order numerical schemes for wave propagation phenomena, International Journal for Numerical Methods in Engineering 45, 1849 (1999)

[53] M. R. Visbal and D. V. Gaitonde, High-order-accurate methods for complex unsteady subsonic flows, AIAA Journal 37, 1231 (1999).

[54] C. T. Jacobs, M. Zauner, N. De Tullio, S. P. Jammy, D. J. Lusher, and N. D. Sandham, An error indicator for finite difference methods using spectral techniques with application to aerofoil simulation, Computers \& Fluids 168, 67 (2018).

[55] M. Zauner, C. T. Jacobs, and N. D. Sandham, Grid refinement using spectral error indicators with application to airfoil DNS, in ECCM-ECFD Conference (Glasgow, 2018).

[56] Á. Tanarro, F. Mallor, N. Offermans, A. Peplinski, R. Vinuesa, and P. Schlatter, Enabling Adaptive Mesh Refinement for Spectral-Element Simulations of Turbulence Around Wing Sections, Flow, Turbulence and Combustion 10.1007/s10494-020-00152-y (2020).

[57] A. Sansica, N. D. Sandham, and Z. Hu, Instability and low-frequency unsteadiness in a shockinduced laminar separation bubble, Journal of Fluid Mechanics 798, 5 (2016).

[58] A. Lozano-Durán and J. Jiménez, Effect of the computational domain on direct simulations of turbulent channels up to $\operatorname{Re} \tau=4200$, Physics of Fluids 26, 011702 (2014).

[59] https://doi.org/10.5258/SOTON/D1484, .

[60] A. Busemann, The Drag Problem at High Supersonic Speeds, Journal of the Aeronautical Sciences 16, 337 (1949), 
[61] M. Zauner, N. D. Sandham, A. P. S. Wheeler, and R. D. Sandberg, Linear Stability Prediction of Vortex Structures on High Pressure Turbine Blades, International Journal of Propulsion and Power 2, 10.3390/ijtpp2020008 (2017).

[62] A. P. S. Wheeler, R. D. Sandberg, N. D. Sandham, R. Pichler, V. Michelassi, and G. Laskowski, Direct Numerical Simulations of a High-Pressure Turbine Vane, Journal of Turbomachinery 138, $10.1115 / 1.4032435(2016)$.

[63] A. K. M. F. Hussain, Coherent structures and turbulence, Journal of Fluid Mechanics 173, 303 (1986).

[64] W. Hu, S. Hickel, and B. van Oudheusden, Dynamics of a supersonic transitional flow over a backward-facing step, Physical Review Fluids 4, 103904 (2019).

[65] Y. Sugioka, D. Numata, K. Asai, S. Koike, K. Nakakita, and T. Nakajima, Polymer/Ceramic Pressure-Sensitive Paint with Reduced Roughness for Unsteady Measurement in Transonic Flow, AIAA Journal 56, 2145 (2018).

[66] A. E. Winkelman and J. B. Barlow, Flowfield Model for a Rectangular Planform Wing beyond Stall, AIAA Journal 18, 1006 (1980).

[67] F. Plante and J. Dandois, Similitude between 3D cellular patterns in transonic buffet and subsonic stall, in AIAA SciTech 2019 Forum (2019).

[68] H.-w. Liepmann and H. Ashkenast, Shock-Wave Oscillations in Wind Tunnels, Journal of the Aeronautical Sciences 14, 295 (1947).

[69] B. Lee and L. H. Ohman, Unsteady Pressure and Force Measurements Associated with Transonic Buffeting of a Two-Dimensional Supercritical Airfoil, Tech. Rep. (National Aeronautical Establishment, 1983).

[70] K. Finke, Shock Oscillations in Transonic Flows and their Prevention, in Symposium Transsonicum II (Springer Berlin Heidelberg, Berlin, Heidelberg, 1976) pp. 57-65.

[71] E. Paladini, O. Marquet, D. Sipp, J. C. Robinet, and J. Dandois, Various approaches to determine active regions in an unstable global mode: Application to transonic buffet, Journal of Fluid Mechanics 881, 617 (2019).

[72] https://www.youtube.com/playlist?list=PLoPRsYcneMTKVv5XxRCReOfhQoDZ2q7GO. 